TRANSACTIONS OF THE

AMERICAN MATHEMATICAL SOCIETY

Volume 354, Number 10, Pages 4067-4084

S 0002-9947(02)03062-3

Article electronically published on June 4, 2002

\title{
THE DYNAMICS OF EXPANSIVE INVERTIBLE ONESIDED CELLULAR AUTOMATA
}

\author{
MASAKAZU NASU
}

\begin{abstract}
Using textile systems, we prove the conjecture of Boyle and Maass that the dynamical system defined by an expansive invertible onesided cellular automaton is topologically conjugate to a topological Markov shift. We also study expansive leftmost-permutive onesided cellular automata and bipermutive endomorphisms of mixing topological Markov shifts.
\end{abstract}

\section{INTRODUCTION}

Let $A$ be the alphabet of $N$ symbols. Let $A^{\mathbf{N}}$ be endowed with a metric compatible with the product topology of the discrete topology on $A$. Let $k \in \mathbf{N}$. Let $f: A^{k+1} \rightarrow A$ be a mapping. Let $\tilde{\varphi}_{f}: A^{\mathbf{N}} \rightarrow A^{\mathbf{N}}$ be the mapping defined as follows: for $\left(a_{i}\right)_{i \in \mathbf{N}} \in A^{\mathbf{N}}, a_{i} \in A$,

$$
\tilde{\varphi}_{f}\left(\left(a_{i}\right)_{i \in \mathbf{N}}\right)=\left(f\left(a_{i} \ldots a_{i+k}\right)\right)_{i \in \mathbf{N}} .
$$

The map $\tilde{\varphi}_{f}$ is the onesided cellular automaton map defined by a local rule $f$. In [BM], Boyle and Maass have proved the following theorem.

Theorem 1.1 (Boyle and Maass $[\mathrm{BM}])$. If the dynamical system $\left(A^{\mathbf{N}}, \tilde{\varphi}_{f}\right)$ is topologically conjugate to a sofic system, then it is topologically conjugate to a topological Markov shift which is shift equivalent to some full $J$-shift, where $J$ and $N$ are divisible by the same primes.

They have also given the following conjecture.

Conjecture 1.2 (Boyle and Maass $[\mathrm{BM}]$ ). If $\tilde{\varphi}_{f}$ is an expansive homeomorphism, then the dynamical system $\left(A^{\mathbf{N}}, \tilde{\varphi}_{f}\right)$ is topologically conjugate to a topological Markov shift.

In this paper, we shall establish a property of a certain class of textile systems (Proposition 3.5), and using it we shall prove

Theorem 1.3. The conjecture of Boyle and Maass is true.

Boyle and Maass have also given two more conjectures in $[\mathrm{BM}]$; one combined with Theorem 1.3 is such that if $\tilde{\varphi}_{f}$ is an expansive homeomorphism, then $\left(A^{\mathbf{N}}, \tilde{\varphi}_{f}\right)$ is topologically conjugate to a full shift, and the other combined with Theorem 1.3,

Received by the editors October 12, 2001 and, in revised form, March 28, 2002.

2000 Mathematics Subject Classification. Primary 37B15; Secondary 37B10, 54H20.

Key words and phrases. Cellular automata, topological Markov shifts, expansive, symbolic dynamics, textile systems.

This research was partially supported by Grant-in-Aid for Scientific Research (No. 11674021), Ministry of Education, Culture, Sports, Science and Technology, Japan. 
in particular, implies that under the same hypothesis, $N$ is divisible by $p^{2}$ for every prime $p$ dividing $N$. We cannot prove these.

A local rule $f: A^{k+1} \rightarrow A$ is said to be leftmost-permutive if for any distinct $a, a^{\prime} \in A$ and any $w \in A^{k}, f(a w)$ and $f\left(a^{\prime} w\right)$ are different.

It is clear that if the onesided cellular automaton map $\tilde{\varphi_{f}}$ defined by a local rule $f$ is injective, then $f$ must be leftmost-permutive. We see that every expansive leftmost-permutive onesided cellular automaton map is necessarily accompanied by expansive invertible onesided cellular automaton maps with the "same" dynamics as it has (Proposition 5.4), so that Theorem 1.1 together with Theorem 1.3 is generalized to expansive leftmost-permutive onesided cellular automata (Theorem 5.5). We also add the result that if a mixing topological Markov shift has a noninvertible bipermutive endomorphism, then the shift must be topologically conjugate to some full shift (Proposition 6.1), which shows that unknown examples mentioned in [N2] p. 52] actually do not exist.

In [N4], the following question was asked. If $\tau$ is a positively expansive continuous map of a compact metric space $X$ onto itself and has the pseudo-orbit tracing property and if $\varphi$ is an expansive homeomorphism with $\varphi \tau=\tau \varphi$, then does $\varphi$ have the pseudo-orbit tracing property? In view of Theorem 3.12(2) of [N2] (a onesided topological Markov shift having an expansive automorphism every power of which is topologically transitive is topologically conjugate to some onesided full shift), Theorem 1.3 answers the question for the case where $X$ is 0 -dimensional in the affirmative under the condition that $\varphi^{n}$ is topologically transitive for all $n \in \mathbf{N}$.

The reader is referred to $[\mathrm{BM}]$ for more information on the subject of this paper, to [Ki] or [LM] for a comprehensive introduction to symbolic dynamics, and to [AH] for information on topological dynamics.

\section{Preliminaries}

We follow the notation and terminology of $\mathbb{N 2}$.

Let $A$ be an alphabet (i.e., a finite nonempty set of symbols). Let $A^{\mathbf{Z}}$ be endowed with the metric $d$ such that for $x=\left(a_{j}\right)_{j \in \mathbf{Z}}$ and $y=\left(b_{j}\right)_{j \in \mathbf{Z}}$ with $a_{j}, b_{j} \in A$, $d(x, y)=0$ if $x=y$, and otherwise, $d(x, y)=1 /(1+k)$, where $k=\min \left\{|j| \mid a_{j} \neq\right.$ $\left.b_{j}\right\}$. The metric $d$ is compatible with the product topology of the discrete topology on $A$. We also endow $A^{\mathbf{N}}$ with a similar metric.

Let $\sigma_{A}: A^{\mathbf{Z}} \rightarrow A^{\mathbf{Z}}$ be defined by $\sigma_{A}\left(\left(a_{j}\right)_{j \in \mathbf{Z}}\right)=\left(a_{j+1}\right)_{j \in \mathbf{Z}}$. The dynamical system $\left(A^{\mathbf{Z}}, \sigma_{A}\right)$ is called the full shift over $A$ or the full $N$-shift if the cardinality of $A$ is $N$. For a closed subset $X$ of $A^{\mathbf{Z}}$ with $\sigma(X)=X$, the dynamical system $\left(X, \sigma_{X}\right)$ is called a subshift over A, where $\sigma_{X}$ is the restriction of $\sigma_{A}$ on $X$.

Let $\tilde{\sigma}_{A}: A^{\mathbf{N}} \rightarrow A^{\mathbf{N}}$ be defined by $\tilde{\sigma}_{A}\left(\left(a_{i}\right)_{i \in \mathbf{N}}\right)=\left(a_{i+1}\right)_{i \in \mathbf{N}}$. The dynamical system $\left(A^{\mathbf{N}}, \tilde{\sigma}_{A}\right)$ is called the onesided full shift over $A$. For a closed subset $\tilde{X}$ of $A^{\mathbf{N}}$ with $\tilde{\sigma}(\tilde{X})=\tilde{X}$, the dynamical system $\left(\tilde{X}, \tilde{\sigma}_{\tilde{X}}\right)$ is called a onesided subshift over $A$, where $\tilde{\sigma}_{\tilde{X}}$ is the restriction of $\tilde{\sigma}_{A}$ on $\tilde{X}$.

Let $G$ be a (directed) graph which may have multiple (directed) arcs and loops. It is represented by

$$
V_{G} \stackrel{i_{G}}{\longleftarrow} A_{G} \stackrel{t_{G}}{\longrightarrow} V_{G},
$$

where $A_{G}$ and $V_{G}$ are the arc-set and the vertex-set, $i_{G}$ and $t_{G}$ are the mappings such that for each $a \in A_{G}, i_{G}(a)$ and $t_{G}(a)$ are the initial and terminal vertices of 
$a$, respectively. Let

$$
X_{G}=\left\{\left(a_{j}\right)_{j \in \mathbf{Z}} \mid \forall j, a_{j} \in A_{G}, t_{G}\left(a_{j}\right)=i_{G}\left(a_{j+1}\right)\right\}
$$

and let

$$
\tilde{X}_{G}=\left\{\left(a_{j}\right)_{j \in \mathbf{N}} \mid \exists\left(a_{j}\right)_{j \in \mathbf{Z}} \in X_{G}\right\} .
$$

Then the subshift $\left(X_{G}, \sigma_{G}\right)$ is called a topological Markov shift defined by $G$. We call the onesided subshift $\left(\tilde{X}_{G}, \tilde{\sigma}_{G}\right)=\left(\tilde{X}_{G}, \tilde{\sigma}_{\tilde{X}_{G}}\right)$ the onesided topological Markov shift defined by $G$.

Let $n \geq 1$. Let $L_{n}(G)$ denote the set of all paths of length $n$ in $G$, that is,

$$
L_{n}(G)=\left\{a_{1} \ldots a_{n} \mid a_{j} \in A_{G}, t_{G}\left(a_{j}\right)=i_{G}\left(a_{j+1}\right) \text { for } j=1, \ldots, n-1\right\} .
$$

For $n \geq 2$, let $G^{[n]}$ be the graph defined as follows: $A_{G^{[n]}}=L_{n}(G), V_{G^{[n]}}=$ $L_{n-1}(G)$, and let $i_{G^{[n]}}$ and $t_{G^{[n]}}$ be the mappings such that for $w=a_{1} \ldots a_{n} \in$ $A_{G^{[n]}}, a_{j} \in A_{G}, i_{G^{[n]}}(w)=a_{1} \ldots a_{n-1}$ and $t_{G^{[n]}}(w)=a_{2} \ldots a_{n}$. We define $G^{[1]}=G$. We call $G^{[n]}$ the higher block graph of order $n$ of $G$.

A $\lambda$-graph $\mathcal{G}$ over an alphabet $A$ is a pair $(G, \lambda)$ of a graph $G$, which is called the support of $\mathcal{G}$, and an onto mapping $\lambda: A_{G} \rightarrow A$. For a $\lambda$-graph $\mathcal{G}=(G, \lambda)$, let

$$
X_{\mathcal{G}}=\left\{\left(\lambda\left(a_{j}\right)\right)_{j \in \mathbf{Z}} \mid\left(a_{j}\right)_{j \in \mathbf{Z}} \in X_{G}\right\} .
$$

Then we have a subshift $\left(X_{\mathcal{G}}, \sigma_{\mathcal{G}}\right)$, which is the sofic system defined by $\mathcal{G}$. We also have the 1-block map $\pi: X_{G} \rightarrow X_{\mathcal{G}}$ defined by $\left(a_{j}\right)_{j \in \mathbf{Z}} \mapsto\left(\lambda\left(a_{j}\right)\right)_{j \in \mathbf{Z}}$, which is called the sofic cover defined by $\mathcal{G}$.

Let $n \geq 1$. For a $\lambda$-graph $\mathcal{G}=(G, \lambda)$, the higher block $\lambda$-graph of order $n$ is defined as $\mathcal{G}^{[n]}=\left(G^{[n]}, \lambda^{[n]}\right)$, where $\lambda^{[n]}$ is the onto mapping such that $\lambda^{[n]}\left(a_{1} \ldots a_{n}\right)=$ $\lambda\left(a_{1}\right) \ldots \lambda\left(a_{n}\right)$ for $a_{1} \ldots a_{n} \in A_{G^{[n]}}, a_{j} \in A_{G}$.

The following is a key lemma for our proof of Theorem 1.3, which is an easy consequence of well-known fundamental results in symbolic dynamics.

Lemma 2.1. Let $\mathcal{G}_{i}=\left(G, \lambda_{i}\right), i=1,2$, be two $\lambda$-graphs over an alphabet $A$ with the same irreducible support $G$. Let $\pi_{i}: X_{G} \rightarrow X_{\mathcal{G}_{i}}$ be the sofic cover defined by $\mathcal{G}_{i}$ for $i=1,2$. If both $\pi_{1}$ and $\pi_{2}$ are bounded-to-one and $\pi_{1}\left(X_{G}\right) \subset \pi_{2}\left(X_{G}\right)$, then $\pi_{1}\left(X_{G}\right)=\pi_{2}\left(X_{G}\right)$.

Proof. Assume that $\pi_{1}\left(X_{G}\right) \subsetneq \pi_{2}\left(X_{G}\right)$. Then there is a word $w$ over $A$ which appears on a point (bisequence) of $\pi_{2}\left(X_{G}\right)=X_{\mathcal{G}_{2}}$ but does not appear on any point of $\pi_{1}\left(X_{G}\right)=X_{\mathcal{G}_{1}}$. Let $l$ be the length of $w$. Consider the higher block $\lambda$-graphs $\mathcal{G}_{i}^{[l]}=\left(G^{[l]}, \lambda_{i}^{[l]}\right)$ of order $l$ of $\mathcal{G}_{i}=\left(G, \lambda_{i}\right)$ for $i=1,2$. Let $\mathcal{H}=\left(H, \lambda_{\mathcal{H}}\right)$ be the $\lambda$-graph obtained from $\mathcal{G}_{2}^{[l]}$ by deleting all arcs having $w$ as their $\lambda_{2}^{[l]}$-labels. Then we have

$$
h\left(\sigma_{H}\right)<h\left(\sigma_{G^{[l]}}\right)=h\left(\sigma_{G}\right)
$$

and

$$
X_{\mathcal{G}_{1}^{[l]}} \subset X_{\mathcal{H}} \subset X_{\mathcal{G}_{2}^{[l]}}
$$

where $h$ denotes topological entropy. By (2.2), we have

$$
h\left(\sigma_{\mathcal{G}_{1}}\right)=h\left(\sigma_{\mathcal{G}_{1}^{[l]}}\right) \leq h\left(\sigma_{\mathcal{H}}\right) \leq h\left(\sigma_{\mathcal{G}_{2}}^{[l]}\right)=h\left(\sigma_{\mathcal{G}_{2}}\right) .
$$


Since $\pi_{1}$ and $\pi_{2}$ are bounded-to-one sofic covers, it follows that $h\left(\sigma_{\mathcal{G}_{i}}\right)=h\left(\sigma_{G}\right)$ for $i=1,2$ and $h\left(\sigma_{\mathcal{H}}\right)=h\left(\sigma_{H}\right)$. Hence we have $h\left(\sigma_{G}\right)=h\left(\sigma_{H}\right)$, which contradicts $(2.1)$.

Now we recall the notion of a textile system introduced in [N2]. A graphhomomorphism $p$ of a graph

$$
\Gamma: \quad V_{\Gamma} \stackrel{i_{\Gamma}}{\longleftarrow} A_{\Gamma} \stackrel{t_{\Gamma}}{\longrightarrow} V_{\Gamma}
$$

into a graph

$$
G: \quad V_{G} \stackrel{i_{G}}{\longleftarrow} A_{G} \stackrel{t_{G}}{\longrightarrow} V_{G}
$$

is defined to be a pair of mappings $p_{A}: A_{\Gamma} \rightarrow A_{G}$ and $p_{V}: V_{\Gamma} \rightarrow V_{G}$ such that the diagram

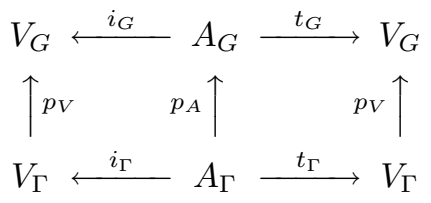

commutes. We write $p: \Gamma \rightarrow G$ to denote it. We will often use $p$ to denote $p_{A}$.

A textile system $T$ over a graph $G$ is defined to be an ordered pair of graphhomomorphisms $p: \Gamma \rightarrow G$ and $q: \Gamma \rightarrow G$ such that each $\alpha \in A_{\Gamma}$ is uniquely determined by the quadruple $\left(i_{\Gamma}(\alpha), t_{\Gamma}(\alpha), p_{A}(\alpha), q_{A}(\alpha)\right)$. We write

$$
T=(p, q: \Gamma \rightarrow G)
$$

We have the following commutative diagram.

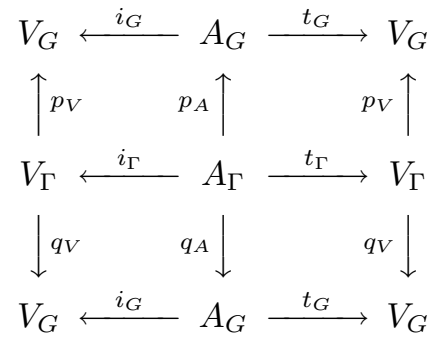

If we observe this diagram vertically, then we have the ordered pair of graph homomorphisms

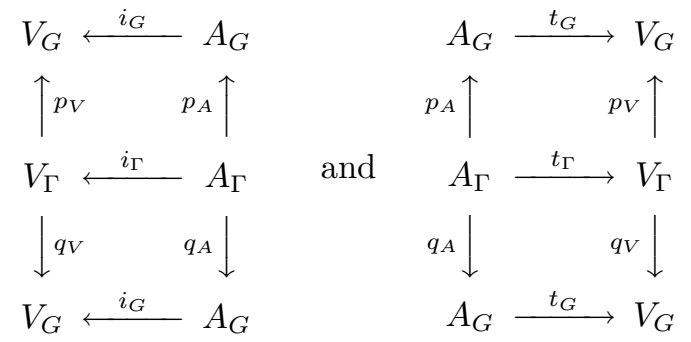


of the graph

$$
\begin{gathered}
A_{G} \\
p_{A} \uparrow \\
A_{\Gamma} \\
q_{A} \downarrow \\
A_{G}
\end{gathered}
$$

into the graph

$$
\begin{gathered}
V_{G} \\
p_{V} \uparrow \\
V_{\Gamma} \\
q_{V} \downarrow \\
V_{G}
\end{gathered}
$$

which gives the definition of the dual $T^{*}$ of the textile system $T$.

Let $T=(p, q: \Gamma \rightarrow G)$ be a textile system. Let $\xi: X_{\Gamma} \rightarrow X_{G}$ and $\eta: X_{\Gamma} \rightarrow X_{G}$ be the 1-block maps given by $p$ and $q$, respectively, that is,

$$
\xi\left(\left(\alpha_{j}\right)_{j \in \mathbf{Z}}\right)=\left(p\left(\alpha_{j}\right)\right)_{j \in \mathbf{Z}}, \quad \eta\left(\left(\alpha_{j}\right)_{j \in \mathbf{Z}}\right)=\left(q\left(\alpha_{j}\right)\right)_{j \in \mathbf{Z}}, \quad\left(\alpha_{j}\right)_{j \in \mathbf{Z}} \in X_{\Gamma}, \alpha_{j} \in A_{\Gamma} .
$$

We say that $T$ is nondegenerate if both $\xi$ and $\eta$ are onto. We also say that $T$ is upwardly nondegenerate if $\eta$ is onto.

Assume that $T$ is upwardly nondegenerate. Let $X_{n}$ and $Z_{n}, n \geq 0$, be defined as follows:

$$
\begin{array}{ll}
X_{0}=X_{G}, & Z_{0}=X_{\Gamma}, \\
X_{n}=\xi\left(Z_{n-1}\right), & Z_{n}=\eta^{-1}\left(X_{n}\right) \quad n=1,2 \ldots
\end{array}
$$

Then by induction on $n$, we have

$$
X_{n} \supset X_{n+1}, \quad Z_{n} \supset Z_{n+1} \quad n=0,1, \ldots
$$

Define

$$
X_{T}=\bigcap_{n=0}^{\infty} X_{n} \text { and } Z_{T}=\bigcap_{n=0}^{\infty} Z_{n} .
$$

We call the subshift $\left(X_{T}, \sigma_{T}\right)$ the woof shift of $T$. The woof shift of the dual $T^{*}$ of $T$ is called the warp shift of $T$. Let $\xi_{T}: Z_{T} \rightarrow X_{T}$ and $\eta_{T}: Z_{T} \rightarrow X_{T}$ be the restrictions of $\xi$ and $\eta$, respectively. We say that $T$ is onesided $1-1$ if $\xi_{T}$ is $1-1$. If $T$ is onesided 1-1, then we define an endomorphism $\varphi_{T}$ of the woof shift $\left(X_{T}, \sigma_{T}\right)$ by $\varphi_{T}=\eta_{T} \xi_{T}^{-1}$.

Let $n \geq 1$. We call the subshift $\left(X_{n}, \sigma_{n}\right)$ the woof shift in height $n$ of $T$. Let $\xi_{n}: Z_{n-1} \rightarrow X_{n}$ be defined by the restriction of $\xi$. We say that $T$ is onesided $1-1$ in height $n$ if $\xi_{n}$ is 1-1. We say that $T$ is onesided bounded-to-one in height $n$ if $\xi_{n}$ is bounded-to-one. We also say that $T$ is saturated in height $n$ if $X_{n}=X_{T}$.

Further we say that $T$ is finitely onesided $1-1$ if there is $n \geq 1$ such that $\xi_{n}$ is 1-1. We also say that $T$ is finitely saturated if there is $n \geq 0$ such that $X_{n}=X_{T}$. 
For $n \in \mathbf{N}$, a 2-dimensional configuration $\left(\alpha_{i, j}\right)_{1 \leq i \leq n, j \in \mathbf{Z}}$ of $\operatorname{arcs} \alpha_{i, j} \in A_{\Gamma}$ is called an obi of width $n$ woven by $T$ if $\left(\alpha_{i, j}\right)_{j \in \mathbf{Z}} \in X_{\Gamma}$ for all $1 \leq i \leq n$ and $\eta\left(\left(\alpha_{i, j}\right)_{j \in \mathbf{Z}}\right)=\xi\left(\left(\alpha_{i+1, j}\right)_{j \in \mathbf{Z}}\right)$ for all $1 \leq i \leq n-1$. The definition of a half-textile

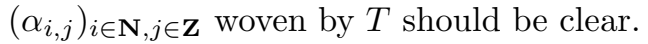

We define $\tilde{X}_{T}=\left\{\left(a_{j}\right)_{j \in \mathbf{N}} \mid \exists\left(a_{j}\right)_{j \in \mathbf{Z}} \in X_{T}\right\}$ and $\tilde{Z}_{T}=\left\{\left(\alpha_{j}\right)_{j \in \mathbf{N}} \mid \exists\left(\alpha_{j}\right)_{j \in \mathbf{Z}} \in\right.$ $\left.Z_{T}\right\}$, and let $\tilde{\xi}_{T}: \tilde{Z}_{T} \rightarrow \tilde{X}_{T}$ and $\tilde{\eta}_{T}: \tilde{Z}_{T} \rightarrow \tilde{X}_{T}$ be defined by $\tilde{\xi}_{T}\left(\left(\alpha_{j}\right)_{j \in \mathbf{N}}\right)=$ $\left(p\left(\alpha_{j}\right)\right)_{j \in \mathbf{N}}$ and $\tilde{\eta}_{T}\left(\left(\alpha_{j}\right)_{j \in \mathbf{N}}\right)=\left(q\left(\alpha_{j}\right)\right)_{j \in \mathbf{N}}$. If $\tilde{\xi}_{T}$ is $1-1$, then we have an endomorphism $\tilde{\varphi}_{T}$ of the onesided woof shift $\left(\tilde{X}_{T}, \tilde{\sigma}_{T}\right)=\left(\tilde{X}_{T}, \tilde{\sigma}_{\tilde{X}_{T}}\right)$ by $\tilde{\varphi}_{T}=\tilde{\eta}_{T} \tilde{\xi}_{T}^{-1}$.

\section{ONESIDED 1-1 $q$-BIRESOLVING TEXTILE SYSTEMS}

Proposition 3.1. Let $T$ be an upwardly nondegenerate textile system. Then $T$ is onesided 1-1 if and only if $T$ is finitely onesided 1-1.

Proof. We follow the notation of Section 2. We first note that for $z \in X_{\Gamma}$ and $n \geq 1, z \in Z_{n-1}$ if and only if $z$ is the uppermost sub-obi $\left(\alpha_{1, j}\right)_{j \in \mathbf{Z}}$ of width 1 of some obi $\left(\alpha_{i, j}\right)_{1 \leq i \leq n, j \in \mathbf{Z}}$ of width $n$ woven by $T$, and $z \in Z_{T}$ if and only if $z$ is the uppermost sub-obi $\left(\alpha_{1, j}\right)_{j \in \mathbf{Z}}$ of width 1 of some half textile $\left(\alpha_{i, j}\right)_{i \in \mathbf{N}, j \in \mathbf{Z}}$ woven by $T$.

By definition the "if" part holds. To prove the converse, suppose that $T$ is not finitely onesided 1-1. Then for each $n \in \mathbf{N}, \xi_{n}$ is not $1-1$. Hence for each $n \in \mathbf{N}$, there exists a pair of obis $\left(\alpha_{i, j}^{(n)}\right)_{1 \leq i \leq n, j \in \mathbf{Z}}$ and $\left(\beta_{i, j}^{(n)}\right)_{1 \leq i \leq n, j \in \mathbf{Z}}$ of width $n$ woven by $T$ such that

$$
\xi\left(\left(\alpha_{1, j}^{(n)}\right)_{j \in \mathbf{Z}}\right)=\xi\left(\left(\beta_{1, j}^{(n)}\right)_{j \in \mathbf{Z}}\right) \quad \text { and } \quad \alpha_{1,0}^{(n)} \neq \beta_{1,0}^{(n)} .
$$

Using a standard compactness argument on the product space $\left(A_{\Gamma}^{\mathbf{Z}}\right)^{\mathbf{N}} \times\left(A_{\Gamma}^{\mathbf{Z}}\right)^{\mathbf{N}}$, we see that there exists a pair of half-textiles $\left(\alpha_{i, j}\right)_{i \in \mathbf{N}, j \in \mathbf{Z}}$ and $\left(\beta_{i, j}\right)_{i \in \mathbf{N}, j \in \mathbf{Z}}$ woven by $T$ with $\xi\left(\left(\alpha_{1, j}\right)_{j \in \mathbf{Z}}\right)=\xi\left(\left(\beta_{1, j}\right)_{j \in \mathbf{Z}}\right)$ and $\alpha_{1,0} \neq \beta_{1,0}$. This implies that $\xi_{T}$ is not $1-1$.

A graph $G$ is said to be nondegenerate if both $i_{G}$ and $t_{G}$ are onto. A graphhomomorphism $h: \Gamma \rightarrow G$ is said to be onto if both $h_{A}$ and $h_{V}$ are onto.

An onto graph-homomorphism $q: \Gamma \rightarrow G$ between nondegenerate graphs is said to be right resolving if for each $u \in V_{\Gamma}$, the restriction of $q_{A}$ on $i_{\Gamma}^{-1}(\{u\})$ is a bijection onto $i_{G}^{-1}\left(\left\{q_{V}(u)\right\}\right)$. It is said to be left resolving if for each $u \in V_{\Gamma}$, the restriction of $q_{A}$ on $t_{\Gamma}^{-1}(\{u\})$ is a bijection onto $t_{G}^{-1}\left(\left\{q_{V}(u)\right\}\right)$. It is said to be biresolving if it is both right resolving and left resolving. If $q: \Gamma \rightarrow G$ is a right resolving or left resolving graph-homomorphism, then the 1-block map $\eta: X_{\Gamma} \rightarrow X_{G}$ given by $q$ is onto.

Proposition 3.2. Let $T=(p, q: \Gamma \rightarrow G)$ be a textile system with $q$ biresolving. If $T$ is onesided bounded-to-one in height $n+1$ with $n \geq 0$ and the woof shift in height $n$ of $T$ is topologically transitive, then $T$ is saturated in height $n$.

Proof. We follow the notation of Section 2.

Since the woof shift $\left(X_{n}, \sigma_{n}\right)$ in height $n$ of $T$ is a topologically transitive sofic system (see [N2, pp. 20-21] or the paragraph preceding Proposition 3.3 below), there exists a $\lambda$-graph $\mathcal{D}=\left(D, \lambda_{\mathcal{D}}\right)$ such that $D$ is an irreducible graph, the sofic system $\left(X_{\mathcal{D}}, \sigma_{\mathcal{D}}\right)$ defined by $\mathcal{D}$ is equal to $\left(X_{n}, \sigma_{n}\right)$, and the sofic cover $\pi_{\mathcal{D}}$ of $\mathcal{D}$ is bounded-to-one. In fact, we can take the right or left Fischer $\lambda$-graph of $\left(X_{n}, \sigma_{n}\right)$ as $\mathcal{D}[\mathrm{F}]$. 
We define a textile system $\hat{T}=(\hat{p}, \hat{q}: \hat{\Gamma} \rightarrow G)$ using $\mathcal{D}=\left(D, \lambda_{\mathcal{D}}\right)$ and $T=(p, q:$ $\Gamma \rightarrow G)$ by

$$
\begin{gathered}
A_{\hat{\Gamma}}=\left\{(\delta, \alpha) \in A_{D} \times A_{\Gamma} \mid \lambda_{\mathcal{D}}(\delta)=q(\alpha)\right\} \\
V_{\hat{\Gamma}}=\left\{\left(i_{D}(\delta), i_{\Gamma}(\alpha)\right) \mid(\delta, \alpha) \in A_{\hat{\Gamma}}\right\}
\end{gathered}
$$

and

$$
\begin{aligned}
& i_{\hat{\Gamma}}((\delta, \alpha))=\left(i_{D}(\delta), i_{\Gamma}(\alpha)\right) \quad t_{\hat{\Gamma}}((\delta, \alpha))=\left(t_{D}(\delta), t_{\Gamma}(\alpha)\right) \\
& \hat{p}((\delta, \alpha))=p(\alpha) \quad \hat{q}((\delta, \alpha))=q(\alpha) \quad \text { for }(\delta, \alpha) \in A_{\hat{\Gamma}} \text {. }
\end{aligned}
$$

Claim. $\hat{\Gamma}$ is the disjoint union of irreducible graphs.

Since $q$ is biresolving, each $b \in A_{G}$ induces the bijection $Q_{b}: q_{V}^{-1}\left(\left\{i_{G}(b)\right\}\right) \rightarrow$ $q_{V}^{-1}\left(\left\{t_{G}(b)\right\}\right)$ such that $Q_{b}(a)=t_{\Gamma}(\alpha)$ for $a \in q_{V}^{-1}\left(\left\{i_{G}(b)\right\}\right)$, where $\alpha$ is the unique arc in $\Gamma$ with $i_{\Gamma}(\alpha)=a$ and $q(\alpha)=b$. For each $u \in V_{D}$, let

$$
W_{u}=\left\{(u, a) \in V_{\hat{\Gamma}} \mid a \in V_{\Gamma}\right\} .
$$

Let $\delta \in A_{D}$. If $u=i_{D}(\delta), v=t_{D}(\delta)$ and $\lambda_{\mathcal{D}}(\delta)=b$, then we can define a bijection $J_{\delta}: W_{u} \rightarrow W_{v}$ by

$$
J_{\delta}((u, a))=\left(v, Q_{b}(a)\right) .
$$

To prove the claim, it suffices to show that if $\left(\delta_{1}, \alpha_{1}\right) \ldots\left(\delta_{l}, \alpha_{l}\right)$ is a path in $\hat{\Gamma}$ with $\delta_{i} \in A_{D}$ and $\alpha_{i} \in A_{\Gamma}$, then there exists a path going from vertex $\left(t_{D}\left(\delta_{l}\right), t_{\Gamma}\left(\alpha_{l}\right)\right)$ to vertex $\left(i_{D}\left(\delta_{1}\right), i_{\Gamma}\left(\alpha_{1}\right)\right)$ in $\hat{\Gamma}$. Put $i_{D}\left(\delta_{1}\right)=u$ and put $t_{D}\left(\delta_{l}\right)=v$. Then $\delta_{1} \ldots \delta_{l}$ is a path going from $u$ to $v$ in $D$. Since $D$ is irreducible, there exists a path $\delta_{1}^{\prime} \ldots \delta_{m}^{\prime}$ going from $v$ to $u$ in $D$. Then $\delta_{1} \ldots \delta_{l} \delta_{1}^{\prime} \ldots \delta_{m}^{\prime}$ is a cycle in $D$, i.e., a path going from $u$ to itself. Let

$$
J=J_{\delta_{m}^{\prime}} \ldots J_{\delta_{1}^{\prime}} J_{\delta_{l}} \ldots J_{\delta_{1}} .
$$

Then $J$ is a bijection of $W_{u}$ onto itself. There exists $r \in \mathbf{N}$ such that $J^{r}$ is the identity map of $W_{u}$. If $J^{\prime}=J^{r-1} J_{\delta_{m}^{\prime}} \ldots J_{\delta_{1}^{\prime}}$, then $J^{\prime}$ is a bijection of $W_{v}$ onto $W_{u}$ with $J^{\prime}\left(t_{\Gamma}\left(\alpha_{l}\right)\right)=i_{\Gamma}\left(\alpha_{1}\right)$, which implies that there exists a path going from vertex $\left(t_{D}\left(\delta_{l}\right), t_{\Gamma}\left(\alpha_{l}\right)\right)$ to vertex $\left(i_{D}\left(\delta_{1}\right), i_{\Gamma}\left(\alpha_{1}\right)\right)$ in $\hat{\Gamma}$. Hence the claim is proved.

Let $\check{\Gamma}$ be any one of the irreducible components of $\hat{\Gamma}$. Let $\breve{p}$ and $\check{q}$ be the restrictions of $\hat{p}$ and $\hat{q}$, respectively, on $A_{\check{\Gamma}}$. Let $\check{\xi}: X_{\check{\Gamma}} \rightarrow X_{G}$ and $\check{\eta}: X_{\check{\Gamma}} \rightarrow X_{G}$ be the 1-block maps given by $\check{p}$ and $\check{q}$, respectively.

Let $\delta_{1} \ldots \delta_{l}$ be any path in $D$ with $\delta_{i} \in A_{D}$. Then there exists a path of the form $\left(\delta_{1}, \alpha_{1}\right) \ldots\left(\delta_{l}, \alpha_{l}\right)$ with $\alpha_{i} \in A_{\Gamma}$ in $\check{\Gamma}$. For let $(u, a) \in V_{\check{\Gamma}}$ with $u \in V_{D}$ and $a \in V_{\Gamma}$. Since $D$ is an irreducible graph, there exists a path $\delta_{1}^{\prime} \ldots \delta_{m}^{\prime}$ going from $u$ to $i_{D}\left(\delta_{1}\right)$ in $D$. It follows that $q_{V}(a)=i_{G}\left(\lambda_{\mathcal{D}}\left(\delta_{1}^{\prime}\right)\right)$. Therefore, since $q$ is right resolving, there is a path $\alpha_{1}^{\prime} \ldots \alpha_{m}^{\prime} \alpha_{1} \ldots \alpha_{l}$ starting from $a$ with

$$
q\left(\alpha_{1}^{\prime}\right) \ldots q\left(\alpha_{m}^{\prime}\right) q\left(\alpha_{1}\right) \ldots q\left(\alpha_{l}\right)=\lambda_{\mathcal{D}}\left(\delta_{1}^{\prime}\right) \ldots \lambda_{\mathcal{D}}\left(\delta_{m}^{\prime}\right) \lambda_{\mathcal{D}}\left(\delta_{1}\right) \ldots \lambda_{\mathcal{D}}\left(\delta_{l}\right) .
$$

This implies that $\left(\delta_{1}^{\prime}, \alpha_{1}^{\prime}\right) \ldots\left(\delta_{m}^{\prime}, \alpha_{m}^{\prime}\right)\left(\delta_{1}, \alpha_{1}\right) \ldots\left(\delta_{l}, \alpha_{l}\right)$ is a path in $\hat{\Gamma}$. Moreover, it is a path in $\check{\Gamma}$ because it starts from $(u, a) \in V_{\check{\Gamma}}$. Hence $\left(\delta_{1}, \alpha_{1}\right) \ldots\left(\delta_{l}, \alpha_{l}\right)$ is a path in $\check{\Gamma}$.

Therefore, we have

$$
\check{\eta}\left(X_{\check{\Gamma}}\right)=\pi_{\mathcal{D}}\left(X_{D}\right)=X_{n}
$$


Let $\left(\left(\delta_{j}, \alpha_{j}\right)\right)_{j \in \mathbf{Z}} \in X_{\check{\Gamma}}$ with $\delta_{j} \in A_{D}$ and $\alpha_{j} \in A_{\Gamma}$. Then $\left(\alpha_{j}\right)_{j \in \mathbf{Z}} \in Z_{n}$ because

$$
\eta\left(\left(\alpha_{j}\right)_{j \in \mathbf{Z}}\right)=\pi_{\mathcal{D}}\left(\left(\delta_{j}\right)_{j \in \mathbf{Z}}\right) \in X_{\mathcal{D}}=X_{n}
$$

Hence if $\check{\pi}: X_{\check{\Gamma}} \rightarrow X_{\Gamma}$ is the mapping $\left(\left(\delta_{j}, \alpha_{j}\right)\right)_{j \in \mathbf{Z}} \mapsto\left(\alpha_{j}\right)_{j \in \mathbf{Z}}$, then

$$
\begin{gathered}
\check{\pi}\left(X_{\check{\Gamma}}\right) \subset Z_{n}, \\
\check{\xi}=\xi \check{\pi}=\xi_{n+1} \check{\pi} \quad \text { and } \quad \check{\eta}=\eta \check{\pi} .
\end{gathered}
$$

Thus we have

$$
\check{\xi}\left(X_{\check{\Gamma}}\right) \subset \xi\left(Z_{n}\right)=X_{n+1} .
$$

Since $\pi_{\mathcal{D}}$ is bounded-to-one, it follows that $\check{\pi}$ is bounded-to-one. Since $\check{\pi}, \xi_{n+1}$ and $\eta$ are all bounded-to-one, $\check{\xi}$ and $\check{\eta}$ are bounded-to-one. Therefore, noting (3.1) and (3.2) with $X_{n} \supset X_{n+1}$, we apply Lemma 2.1 to the textile system $\check{T}=(\check{p}, \check{q}: \check{\Gamma} \rightarrow$ $G)$. Then we have $X_{n}=X_{n+1}$ and Proposition 3.2 is proved.

Let $T=(p, q: \Gamma \rightarrow G)$ be a textile system with $p$ or $q$ right resolving or left resolving. Let $n \geq 1$. We consider the $n$-th composition power $T^{n}$ of $T$ (see [N2]). This is given as the dual of the $n$-th product power $\left(T^{*}\right)^{(n)}=\left(\left(p^{*}\right)^{(n)},\left(q^{*}\right)^{(n)}\right.$ : $\left.\left(\Gamma^{*}\right)^{n} \rightarrow\left(G^{*}\right)^{n}\right)$ of the dual $T^{*}=\left(p^{*}, q^{*}: \Gamma^{*} \rightarrow G^{*}\right)$ of $T$ such that the graphs $\left(\Gamma^{*}\right)^{n}$ and $\left(G^{*}\right)^{n}$ and the graph-homomorphisms $\left(p^{*}\right)^{(n)}$ and $\left(q^{*}\right)^{(n)}$ are given as follows: $A_{\left(\Gamma^{*}\right)^{n}}=L_{n}\left(\Gamma^{*}\right), V_{\left(\Gamma^{*}\right)^{n}}=V_{\Gamma^{*}}, A_{\left(G^{*}\right)^{n}}=L_{n}\left(G^{*}\right), V_{\left(G^{*}\right)^{n}}=V_{G^{*}}$, and

$$
\begin{aligned}
i_{\left(\Gamma^{*}\right)^{n}}\left(\alpha_{1} \ldots \alpha_{n}\right) & =i_{\Gamma^{*}}\left(\alpha_{1}\right) & t_{\left(\Gamma^{*}\right)^{n}}\left(\alpha_{1} \ldots \alpha_{n}\right) & =t_{\Gamma^{*}}\left(\alpha_{n}\right) \\
i_{\left(G^{*}\right)^{n}}\left(a_{1} \ldots a_{n}\right) & =i_{G^{*}}\left(a_{1}\right) & t_{\left(G^{*}\right)^{n}}\left(a_{1} \ldots a_{n}\right) & =t_{G^{*}}\left(a_{n}\right) \\
\left(p^{*}\right)^{(n)}\left(\alpha_{1} \ldots \alpha_{n}\right) & =p^{*}\left(\alpha_{1}\right) \ldots p^{*}\left(\alpha_{n}\right) & & \left(q^{*}\right)^{(n)}\left(\alpha_{1} \ldots \alpha_{n}\right)=q^{*}\left(\alpha_{1}\right) \ldots q^{*}\left(\alpha_{n}\right),
\end{aligned}
$$

where $\alpha_{1} \ldots \alpha_{n} \in L_{n}\left(\Gamma^{*}\right)$ with $\alpha_{i} \in A_{\Gamma^{*}}$ and $a_{1} \ldots a_{n} \in L_{n}\left(G^{*}\right)$ with $a_{i} \in A_{G^{*}}$. We can put

$$
T^{n}=\left(\left(T^{*}\right)^{(n)}\right)^{*}=(\bar{p}, \bar{q}: \bar{\Gamma} \rightarrow G) .
$$

We note that the vertex-set $V_{\bar{\Gamma}}$ is equal to $L_{n}\left(G^{*}\right)$. If $q$ is biresolving, then $\bar{q}$ is biresolving. We observe that $X_{\bar{\Gamma}}$ is the set of all elements $\left(\left(\alpha_{i, j}\right)_{1 \leq i \leq n}\right)_{j \in \mathbf{Z}}, \alpha_{i, j} \in$ $A_{\Gamma}$, such that $\left(\alpha_{i, j}\right)_{1 \leq i \leq n, j \in \mathbf{Z}}$ is an obi of width $n$ woven by $T$. Hence $X_{\bar{\Gamma}}$ will be identified with the set of all obis of width $n$ woven by $T$. Let $\bar{\xi}: X_{\bar{\Gamma}} \rightarrow X_{G}$ and $\bar{\eta}: X_{\bar{\Gamma}} \rightarrow X_{G}$ be the 1-block maps given by $\bar{p}$ and $\bar{q}$, respectively. If $\bar{z}=$ $\left(\alpha_{i, j}\right)_{1 \leq i \leq n, j \in \mathbf{Z}}$ is in $X_{\bar{\Gamma}}$, then $\bar{\xi}(\bar{z})$ is its uppermost thread $\xi\left(\left(\alpha_{1, j}\right)_{j \in \mathbf{Z}}\right)$ and $\bar{\eta}(\bar{z})$ is its lowermost thread $\eta\left(\left(\alpha_{n, j}\right)_{j \in \mathbf{Z}}\right)$. We have $\bar{\xi}\left(X_{\bar{\Gamma}}\right)=X_{n}$, where $\left(X_{n}, \sigma_{n}\right)$ is the woof shift in height $n$ of $T$. The subshift $\left(X_{n}, \sigma_{n}\right)$ is a sofic system defined by the $\lambda$-graph $(\bar{\Gamma}, \bar{p})$.

Proposition 3.3. Let $T=(p, q: \Gamma \rightarrow G)$ be a textile system with $q$ biresolving, $G$ irreducible and its warp shift $\left(X_{T^{*}}, \sigma_{T^{*}}\right)$ topologically mixing. If $T$ is onesided 1-1 in height $n+1$ with $n \geq 1$, then the woof shift $\left(X_{n}, \sigma_{n}\right)$ in height $n$ of $T$ is topologically transitive. If in addition $G$ is aperiodic, then $\left(X_{n}, \sigma_{n}^{m}\right)$ is topologically transitive for all $m \in \mathbf{N}$.

Proof. We follow the notation above and that of Section 2.

First we observe that $T^{n}=(\bar{p}, \bar{q}: \bar{\Gamma} \rightarrow G)$ is onesided 1-1 in height 2 . Since $T$ is onesided 1-1 in height $n+1$, if $\left(\alpha_{i, j}\right)_{1 \leq i \leq n+1, j \in \mathbf{Z}}$ is an obi of width $n+1$ woven by $T=(p, q: \Gamma \rightarrow G)$ with $\alpha_{i, j} \in A_{\Gamma}$, then $\left(\alpha_{1, j}\right)_{j \in \mathbf{Z}}$ is uniquely determined by $\xi\left(\left(\alpha_{1, j}\right)_{j \in \mathbf{Z}}\right)$, because $\left(\alpha_{1, j}\right)_{j \in \mathbf{Z}} \in Z_{n}$ and $\xi_{n+1}=\xi / Z_{n}$ is $1-1$. Assume that 
$\left(\bar{\alpha}_{i, j}\right)_{1 \leq i \leq 2, j \in \mathbf{Z}}$ is an obi of width 2 woven by $T^{n}$. Then $\bar{\alpha}_{1, j}$ and $\bar{\alpha}_{2, j}$ are of the form $\left(\alpha_{i, j}\right)_{1 \leq i \leq n}$ and $\left(\alpha_{i, j}\right)_{n+1 \leq i \leq 2 n}$, respectively, with $\left(\alpha_{i, j}\right)_{1 \leq i \leq 2 n, j \in \mathbf{Z}}, \alpha_{i, j} \in A_{\Gamma}$, woven by $T$. Since $\left(\alpha_{s+i, j}\right)_{0 \leq i \leq n, j \in \mathbf{Z}}$ is an obi of width $n+1$ for $1 \leq s \leq n$, $\left(\alpha_{s, j}\right)_{j \in \mathbf{Z}}$ is uniquely determined by $\xi\left(\left(\alpha_{s, j}\right)_{j \in \mathbf{Z}}\right)$ for $1 \leq s \leq n$. Hence it follows that $\left(\alpha_{i, j}\right)_{1 \leq i \leq n, j \in \mathbf{Z}}=\left(\bar{\alpha}_{1, j}\right)_{j \in \mathbf{Z}}$ is uniquely determined by $\xi\left(\left(\alpha_{1, j}\right)_{j \in \mathbf{Z}}\right)=\bar{\xi}\left(\left(\bar{\alpha}_{1, j}\right)_{j \in \mathbf{Z}}\right)$. Thus $T^{n}$ is onesided 1-1 in height 2 .

Assume that $\bar{\Gamma}$ is not irreducible. Then, since $\bar{q}$ is biresolving and $G$ is irreducible, $\bar{\Gamma}$ is the disjoint union of at least two irreducible components. (In fact, this follows from the claim in the proof of Proposition 3.2 if we consider $\hat{\Gamma}$ constructed by using the $\lambda$-graph $\left(G, \operatorname{id}_{A_{G}}\right)$ and $\bar{q}: \bar{\Gamma} \rightarrow G$ instead of $\mathcal{D}$ and $q: \Gamma \rightarrow G$.) Let $\breve{\Gamma}$ be one of the components.

Let $\breve{T}=(\breve{p}, \breve{q}: \breve{\Gamma} \rightarrow G)$ be the textile system such that $\breve{p}$ and $\breve{q}$ are the restrictions of $\bar{p}$ and $\bar{q}$ on $\breve{\Gamma}$. Then since $\bar{q}$ is biresolving, so is $\breve{q}$. Since $T^{n}$ is onesided 1-1 in height 2 , so is $\breve{T}$. Since $\breve{\Gamma}$ is an irreducible graph, the woof shift in height 1 of $\breve{T}$ is topologically transitive. Therefore, if we apply Proposition 3.2 to $\breve{T}$, then we see that $\breve{T}$ is saturated in height 1 .

This implies that if $\left(\alpha_{i, j}\right)_{1 \leq i \leq n, j \in \mathbf{Z}}, \alpha_{i, j} \in A_{\Gamma}$, is an obi of width $n$ woven by $T$ such that $\left(\alpha_{i, j}\right)_{1 \leq i \leq n, j \in \mathbf{Z}} \in X_{\breve{\Gamma}}$, then there exists a half-textile $\left(\beta_{i, j}\right)_{i \in \mathbf{N}, j \in \mathbf{Z}}, \beta_{i, j} \in$ $A_{\Gamma}$, woven by $T$ such that

$$
\begin{gathered}
\xi\left(\left(\beta_{1, j}\right)_{j \in \mathbf{Z}}\right)=\xi\left(\left(\alpha_{1, j}\right)_{j \in \mathbf{Z}}\right) \\
\left(\beta_{t n+i, j}\right)_{1 \leq i \leq n, j \in \mathbf{Z}} \in X_{\breve{\Gamma}} \quad \forall t \geq 0 .
\end{gathered}
$$

Let $\bar{u} \in V_{\breve{\Gamma}}$ and let $\bar{v} \in V_{\bar{\Gamma}}-V_{\breve{\Gamma}}$. Since $q$ is biresolving, the dual $T^{*}=\left(p^{*}, q^{*}: \Gamma^{*} \rightarrow\right.$ $\left.G^{*}\right)$ of $T$ is $L L$, that is, $p^{*}$ and $q^{*}$ are left resolving, so that $T^{*}$ is nondegenerate. Hence $\left(X_{T^{*}}, \sigma_{T^{*}}\right)=\left(X_{G^{*}}, \sigma_{G^{*}}\right)$, so that $\bar{u} \in L_{n}\left(G^{*}\right)$ appears on some point in $X_{T^{*}}$ and so does $\bar{v} \in L_{n}\left(G^{*}\right)$. Since $\left(X_{T^{*}}, \sigma_{T^{*}}\right)$ is topologically mixing, there exists a half-textile $\left(\alpha_{i, j}\right)_{i \in \mathbf{N}, j \in \mathbf{Z}}$ woven by $T$ such that

$$
\begin{aligned}
\left(i_{\Gamma}\left(\alpha_{i, 0}\right)\right)_{1 \leq i \leq n} & =\bar{u} \\
\left(i_{\Gamma}\left(\alpha_{t n+i, 0}\right)\right)_{1 \leq i \leq n} & =\bar{v} \quad \exists t \geq 1 .
\end{aligned}
$$

Since $\breve{\Gamma}$ is an irreducible graph, $\left(\alpha_{i, j}\right)_{1 \leq i \leq n, j \in \mathbf{Z}} \in X_{\breve{\Gamma}}$. Therefore, it follows from the above that there exists a half-textile $\left(\beta_{i, j}\right)_{i \in \mathbf{N}, j \in \mathbf{Z}}, \beta_{i, j} \in A_{\Gamma}$, woven by $T$ such that

$$
\begin{gathered}
\xi\left(\left(\beta_{1, j}\right)_{j \in \mathbf{Z}}\right)=\xi\left(\left(\alpha_{1, j}\right)_{j \in \mathbf{Z}}\right) \\
\left(\beta_{t n+i, j}\right)_{1 \leq i \leq n, j \in \mathbf{Z}} \in X_{\breve{\Gamma}} \quad \forall t \geq 0 .
\end{gathered}
$$

Clearly the half-textiles $\left(\alpha_{i, j}\right)_{i \in \mathbf{N}, j \in \mathbf{Z}}$ and $\left(\beta_{i, j}\right)_{i \in \mathbf{N}, j \in \mathbf{Z}}$ are different, but they have the same uppermost thread, which contradicts that $T$ is onesided 1-1. Thus we have proved that $\bar{\Gamma}$ is irreducible. Therefore, $\left(X_{n}, \sigma_{n}\right)$ is topologically transitive.

Assume that $G$ is aperiodic. Let $m \in \mathbf{N}$. Let $T^{(m)}=\left(p^{(m)}, q^{(m)}: \Gamma^{m} \rightarrow\right.$ $\left.G^{m}\right)$ be the $m$-th product power of $T$. Then $q^{(m)}$ is biresolving, $G^{m}$ is irreducible, $\left(X_{\left(T^{(m)}\right)^{*}}, \sigma_{\left(T^{(m))^{*}}\right.}\right)=\left(X_{T^{*}}, \sigma_{T^{*}}\right)$ and $T^{(m)}$ is onesided 1-1 in height $n+1$. Hence it follows from the above that the woof shift of $T^{(m)}$ in height $n$ is topologically transitive. Therefore, $\left(X_{n}, \sigma_{n}^{m}\right)$ is topologically transitive, because it is topologically conjugate to the woof shift of $T^{(m)}$ in height $n$. 
Proposition 3.4 (Proposition 2.1(2) of [N2]). If $T$ is an upwardly nondegenerate, onesided 1-1 and finitely saturated textile system, then $\left(X_{T}, \sigma_{T}\right)$ is topologically conjugate to a topological Markov shift.

Proposition 3.5. If $T=(p, q: \Gamma \rightarrow G)$ is a onesided 1-1 textile system with $q$ biresolving, $G$ irreducible and $\left(X_{T^{*}}, \sigma_{T^{*}}\right)$ topologically mixing, then $\left(X_{T}, \sigma_{T}\right)$ is topologically conjugate to a topologically transitive topological Markov shift. If in addition $G$ is aperiodic, then $\left(X_{T}, \sigma_{T}\right)$ is topologically conjugate to a topologically mixing topological Markov shift.

Proof. The proposition follows from Propositions 3.1, 3.2, 3.3, and 3.4.

Let $T=(p, q: \Gamma \rightarrow G)$ be a textile system. Let $n \in \mathbf{N}$. The higher block system $T^{[n]}$ of order $n$ of $T$ is defined by $T^{[n]}=\left(p^{[n]}, q^{[n]}: \Gamma^{[n]} \rightarrow G^{[n]}\right)$ with $p^{[n]}, q^{[n]}$ such that

$$
p^{[n]}\left(\alpha_{1} \ldots \alpha_{n}\right)=p\left(\alpha_{1}\right) \ldots p\left(\alpha_{n}\right), \quad q^{[n]}\left(\alpha_{1} \ldots \alpha_{n}\right)=q\left(\alpha_{1}\right) \ldots q\left(\alpha_{n}\right)
$$

for $\alpha_{1} \ldots \alpha_{n} \in A_{\Gamma^{[n]}}=L_{n}(\Gamma), \alpha_{i} \in A_{\Gamma}$.

Corollary 3.6. Let $T=(p, q: \Gamma \rightarrow G)$ be a onesided 1-1 textile system with $q$ biresolving, $G$ irreducible and $\left(X_{T^{*}}, \sigma_{T^{*}}\right)$ topologically mixing. Then there exist $n \in \mathbf{N}$ and a onesided 1-1, nondegenerate textile system $T^{\prime}=\left(p^{\prime}, q^{\prime}: \Gamma^{\prime} \rightarrow G^{\prime}\right)$ with $q^{\prime}$ biresolving and $G^{\prime}$ irreducible such that $T^{\prime}$ is a subsystem of $T^{[n]},\left(X_{G^{\prime}}, \sigma_{G^{\prime}}\right)=$ $\left(X_{T^{[n]}}, \sigma_{T^{[n]}}\right)$ and $\varphi_{T^{\prime}}=\varphi_{T^{[n]}}$ (hence there exists a conjugacy $\rho:\left(X_{T}, \sigma_{T}\right) \rightarrow$ $\left(X_{G^{\prime}}, \sigma_{G^{\prime}}\right)$ such that $\left.\varphi_{T}=\rho^{-1} \varphi_{T^{\prime}} \rho\right)$.

Proof. By Proposition 3.5, $\left(X_{T}, \sigma_{T}\right)$ is a topologically transitive subshift of finite type, and so is $\left(Z_{T}, \varsigma_{T}\right)$ because $\xi_{T}$ is $1-1$. Therefore there exists $n \in \mathbf{N}$ such that both $\left(Z_{T^{[n]}}, \varsigma_{T^{[n]}}\right)=\left(Z_{T}^{[n]}, \varsigma_{T}^{[n]}\right)$ and $\left(X_{T^{[n]}}, \sigma_{T^{[n]}}\right)=\left(X_{T}^{[n]}, \sigma_{T}^{[n]}\right)$ are topological Markov shifts. Let $\Gamma^{\prime}$ and $G^{\prime}$ be the irreducible graphs which define $\left(Z_{T^{[n]}}, \varsigma_{T^{[n]}}\right)$ and $\left(X_{T^{[n]}}, \sigma_{T^{[n]}}\right)$, respectively. Let $T^{\prime}=\left(p^{\prime}, q^{\prime}: \Gamma^{\prime} \rightarrow G^{\prime}\right)$ be the subsystem of $T^{[n]}=\left(p^{[n]}, q^{[n]}: \Gamma^{[n]} \rightarrow G^{[n]}\right)$, that is, $p^{\prime}$ and $q^{\prime}$ are the restrictions of $p^{[n]}$ and $q^{[n]}$, respectively, on $\Gamma^{\prime}$. Then $T^{\prime}$ is nondegenerate. Since $\xi_{T^{\prime}}=\xi_{T^{[n]}}, \eta_{T^{\prime}}=\eta_{T^{[n]}}$ and $\xi_{T}$ is $1-1$, we see that $\xi_{T^{\prime}}$ is $1-1$ and $\varphi_{T^{\prime}}=\varphi_{T^{[n]}}$. Since $q^{\prime}$ is a restriction of $q^{[n]}$ and $q^{[n]}$ is biresolving, it follows that $q^{\prime}$ is weakly right resolving (i.e., the restriction of $q_{A}^{\prime}$ on $i_{\Gamma^{\prime}}^{-1}(\{u\})$ is injective for all $\left.u \in V_{\Gamma^{\prime}}\right)$ and weakly left resolving (i.e., the restriction of $q_{A}^{\prime}$ on $t_{\Gamma^{\prime}}^{-1}(\{u\})$ is injective for all $\left.u \in V_{\Gamma^{\prime}}\right)$. The irreducible graphs $G^{\prime}$ and $\Gamma^{\prime}$ have the same spectral radius. It is well known (see [LM, Proposition 8.2.2 ]) that for a graph-homomorphism between irreducible graphs with the same spectral radius, "weakly right resolving" and "right resolving" are the same, and so are "weakly left resolving" and "left resolving". Thus $q$ ' is biresolving.

\section{Proof of Theorem 1.3}

First we restate Theorem 1.3 in an explicit form.

Let $A$ be an alphabet. Let $k \in \mathbf{N}$. Let $f: A^{k+1} \rightarrow A$ and $g: A^{k+1} \rightarrow A$ be mappings. Let $\tilde{\varphi}_{f}: A^{\mathbf{N}} \rightarrow A^{\mathbf{N}}$ and $\tilde{\varphi}_{g}: A^{\mathbf{N}} \rightarrow A^{\mathbf{N}}$ be defined as in Section 1 . Assume that $\tilde{\varphi}_{g}$ is a homeomorphism, and define

$$
\tilde{\varphi}_{f, g}=\tilde{\varphi}_{f}\left(\tilde{\varphi}_{g}\right)^{-1} .
$$

Theorem 1.3. If $\tilde{\varphi}_{f, g}$ is an expansive homeomorphism, then $\left(A^{\mathbf{N}}, \tilde{\varphi}_{f, g}\right)$ is topologically conjugate to a topological Markov shift. 
Let $T^{*}=\left(p^{*}, q^{*}: \Gamma^{*} \rightarrow G_{A}\right)$ be the textile system defined as follows. The graph $G_{A}$ is the 1-vertex graph with arc-set $A$, that is, $A_{G_{A}}=A, V_{G_{A}}$ is a singleton, say $\left\{v_{0}\right\}$, and $i_{G_{A}}(a)=t_{G_{A}}(a)=v_{0}$ for all $a \in A_{G_{A}} . \Gamma^{*}=G_{A}^{[k+1]}$. The graphhomomorphisms $p^{*}: \Gamma^{*} \rightarrow G_{A}$ and $q^{*}: \Gamma^{*} \rightarrow G_{A}$ are defined by

$$
p^{*}(w)=g(w), \quad q^{*}(w)=f(w), \quad w \in A_{\Gamma^{*}}=A^{k+1} .
$$

We note:

(1) $\left(X_{T^{*}}, \sigma_{T^{*}}\right)=\left(A^{\mathbf{Z}}, \sigma_{A}\right)$.

Let $T$ be the dual of $T^{*}$. Let $B=A^{k}$. Then we can write $T=\left(p, q: \Gamma \rightarrow G_{B}\right)$, where $G_{B}$ is the 1-vertex graph with arc-set $B$ and vertex-set $\left\{v_{0}\right\}$. Let $\tilde{\xi}^{*}$ and $\tilde{\eta}^{*}$ be the mappings of $\tilde{X}_{\Gamma^{*}}$ onto $A^{\mathbf{N}}$ defined by $\tilde{\xi}^{*}\left(\left(\alpha_{i}\right)_{i \in \mathbf{N}}\right)=\left(p^{*}\left(\alpha_{i}\right)\right)_{i \in \mathbf{N}}$ and $\tilde{\eta}^{*}\left(\left(\alpha_{i}\right)_{i \in \mathbf{N}}\right)=\left(q^{*}\left(\alpha_{i}\right)\right)_{i \in \mathbf{N}}$, where $\left(\alpha_{i}\right)_{i \in \mathbf{N}} \in \tilde{X}_{\Gamma^{*}}, \alpha_{i} \in A_{\Gamma^{*}}$. Since $\tilde{\varphi}_{f}$ and $\tilde{\varphi}_{g}$ are bijections, so are $\tilde{\xi}^{*}$ and $\tilde{\eta}^{*}$, and $\tilde{\varphi}_{f, g}=\tilde{\eta}^{*}\left(\tilde{\xi}^{*}\right)^{-1}$. Since $f$ and $g$ are leftmostpermutive, $T^{*}$ is LL, i.e., $p^{*}$ and $q^{*}$ are left resolving. Hence

(2) $T=\left(p, q: \Gamma \rightarrow G_{B}\right)$ has the property that $q$ is biresolving.

Since $\tilde{\varphi}_{f, g}$ is expansive with $\tilde{\varphi}_{T^{*}}=\tilde{\varphi}_{f, g}$ and $\left(A^{\mathbf{N}}, \tilde{\varphi}_{f, g}\right)=\left(\tilde{X}_{T^{*}}, \tilde{\varphi}_{T^{*}}\right)$, applying Theorem 2.11 of [N2] to $T^{*}$, we know:

(3) $T$ is onesided $1-1$ and $\left(A^{\mathbf{N}}, \tilde{\varphi}_{f, g}\right)$ is topologically conjugate to $\left(X_{T}, \sigma_{T}\right)$.

Theorem 1.3 is proved by (1),(2),(3) and Proposition 3.5 (or Propositions 3.1, 3.2, 3.3 and Theorem 1.1).

\section{LeftMost-PERMUtive ONESIDED CELlular AUtOMATA}

The following is the dual expression of a refinement of Lemma 3.11 of $\mathrm{N} 2$.

Proposition 5.1. Let $T^{*}=\left(p^{*}, q^{*}: \Gamma^{*} \rightarrow G^{*}\right)$ be a onesided 1-1, LL textile system with dual T. Suppose that $\sigma_{T}^{m}$ is topologically transitive for all $m \in \mathbf{N}$. Then there exist an alphabet $A$ and a onesided 1-1, LL textile system $T_{0}{ }^{*}=\left(p_{0}{ }^{*}, q_{0}{ }^{*}: \Gamma^{*} \rightarrow\right.$ $\left.G_{A}\right)$ with dual $T_{0}$, where $G_{A}$ is the one-vertex graph with $A_{G_{A}}=A$, such that there exists a topological conjugacy $\tilde{\psi}:\left(\tilde{X}_{G^{*}}, \tilde{\sigma}_{G^{*}}\right) \rightarrow\left(A^{\mathbf{N}}, \tilde{\sigma}_{A}\right)$ with $\tilde{\varphi}_{T^{*}}=\tilde{\psi}^{-1} \tilde{\varphi}_{T_{0}^{*}} \tilde{\psi}$, and $\xi_{T_{0}}=\xi_{T}$ and $\eta_{T_{0}}=\eta_{T}$.

Proof. The proof of Lemma 3.10 of [N2] proves that for two left resolving graphhomomorphisms $h: \Gamma^{*} \rightarrow G^{*}$ and $h^{\prime}: \Gamma^{*} \rightarrow G^{*}$ with $G^{*}$ "column reduced", if $h$ gives a conjugacy of $\left(X_{\Gamma^{*}}, \sigma_{\Gamma^{*}}\right)$ onto $\left(X_{G^{*}}, \sigma_{G^{*}}\right)$, then for $u, v \in V_{\Gamma^{*}}, h(u)=h(v)$ implies $h^{\prime}(u)=h^{\prime}(v)$. Using this, the proof of Lemma 3.11 of [N2] with a few obvious modifications proves Proposition 5.1.

Let $\tilde{\varphi}$ be an endomorphism of a onesided topological Markov shift $\left(\tilde{X}_{G^{*}}, \tilde{\sigma}_{G^{*}}\right)$. Then we call $\tilde{\varphi}$ an $L L$ endomorphism of the shift if there exists a onesided 1-1, LL textile system over $G^{*}$ such that $\tilde{\varphi}_{T^{*}}=\tilde{\varphi}$. If $\tilde{\varphi}=\tilde{\varphi}_{f}$ for some mapping (local rule) $f: L_{k+1}\left(G^{*}\right) \rightarrow A_{G^{*}}$ with $k \geq 0$ which induces a left resolving graphhomomorphism of $\left(G^{*}\right)^{[k+1]}$ onto $G^{*}$, then $\tilde{\varphi}$ is called an leftmost-permutive endomorphism of $\left(\tilde{X}_{G^{*}}, \tilde{\sigma}_{G^{*}}\right)$, where $\tilde{\varphi}_{f}$ is defined by

$$
\tilde{\varphi}_{f}\left(\left(a_{i}\right)_{i \in \mathbf{N}}\right)=\left(f\left(a_{i} \ldots a_{i+k}\right)\right)_{i \in \mathbf{N}}, \quad\left(a_{i}\right)_{i \in \mathbf{N}} \in \tilde{X}_{G^{*}}, \quad a_{i} \in A_{G^{*}} .
$$

Remark 5.2. Let $\tilde{\varphi}$ be an endomorphism of a onesided topological Markov shift. Then $\tilde{\varphi}$ is an LL endomorphism of the shift if and only if $\tilde{\varphi}$ is a leftmost-permutive endomorphism of the shift. 
Proof. Let $G^{*}$ be a graph. If a local rule $f: L_{k+1}\left(G^{*}\right) \rightarrow A_{G^{*}}$ with $k \geq 0$ induces a left resolving graph-homomorphism $q^{*}:\left(G^{*}\right)^{[k+1]} \rightarrow G^{*}$, then we have $\tilde{\varphi}_{f}=\tilde{\varphi}_{T^{*}}$ for the onesided 1-1, LL textile system $T^{*}=\left(p^{*}, q^{*}:\left(G^{*}\right)^{[k+1]} \rightarrow G^{*}\right)$, where $p^{*}$ is such that $p^{*}\left(a_{1} \ldots a_{k+1}\right)=a_{1}$ for $a_{1} \ldots a_{k+1} \in A_{\left(G^{*}\right)^{[k+1]}}, a_{i} \in A_{G^{*}}$.

Conversely let $T^{*}=\left(p^{*}, q^{*}: \Gamma^{*} \rightarrow G^{*}\right)$ be a onesided 1-1, LL textile system. Since $\tilde{\xi}^{*}: \tilde{X}_{\Gamma^{*}} \rightarrow \tilde{X}_{G^{*}}$ given by the left resolving graph-homomorphism $p^{*}$ is 1-1, there exists $k \in \mathbf{N}$ such that for any $\alpha \alpha_{1} \ldots \alpha_{k} \in L_{k+1}\left(\Gamma^{*}\right)$ with $\alpha, \alpha_{i} \in A_{\Gamma^{*}}$, the path $p^{*}(\alpha) p^{*}\left(\alpha_{1}\right) \ldots p^{*}\left(\alpha_{k}\right)$ in $G^{*}$ uniquely determines $\alpha$. Hence we can define a local rule $f: L_{k+1}\left(G^{*}\right) \rightarrow A_{G^{*}}$ by

$$
f\left(p^{*}(\alpha) p^{*}\left(\alpha_{1}\right) \ldots p^{*}\left(\alpha_{k}\right)\right)=q^{*}(\alpha) .
$$

Clearly we have $\tilde{\varphi}_{T^{*}}=\tilde{\varphi}_{f}$. Since $p^{*}$ is left resolving, it follows that for any $\alpha_{1} \ldots \alpha_{k} \in L_{k}\left(\Gamma^{*}\right)$ with $\alpha_{i} \in A_{\Gamma^{*}}$, the path $p^{*}\left(\alpha_{1}\right) \ldots p^{*}\left(\alpha_{k}\right)$ uniquely determines $i_{\Gamma^{*}}\left(\alpha_{1}\right)$. Hence we can define a mapping $F: L_{k}\left(G^{*}\right) \rightarrow V_{\Gamma^{*}}$ by $F\left(p^{*}\left(\alpha_{1}\right) \ldots p^{*}\left(\alpha_{k}\right)\right)$ $=i_{\Gamma^{*}}\left(\alpha_{1}\right)$. Let $Q^{*}:\left(G^{*}\right)^{[k+1]} \rightarrow G^{*}$ be the graph-homomorphism induced by $f$. Then $Q_{V}^{*}\left(a_{1} \ldots a_{k}\right)=q_{V}^{*}\left(F\left(a_{1} \ldots a_{k}\right)\right)$ for $a_{1} \ldots a_{k} \in L_{k}\left(G^{*}\right)=V_{\left(G^{*}\right)^{[k+1]}}$. For $a a_{1} \ldots a_{k} \in L_{k+1}\left(G^{*}\right)=A_{\left(G^{*}\right)^{[k+1]}}$, we have $Q_{A}^{*}\left(a a_{1} \ldots a_{k}\right)=q_{A}^{*}(\alpha)$, where $\alpha$ is the unique arc in $\Gamma^{*}$ such that $t_{\Gamma^{*}}(\alpha)=F\left(a_{1} \ldots a_{k}\right)$ and $p_{A}^{*}(\alpha)=a$. Since $q^{*}$ is left resolving, it easily follows that $Q^{*}$ is left resolving.

The following is an extension of a major part of Theorem $3.12(2)$ of [N2].

Corollary 5.3. If $\tilde{\varphi}$ is a leftmost-permutive endomorphism of a onesided topological Markov shift $\left(\tilde{X}_{G^{*}}, \tilde{\sigma}_{G^{*}}\right)$ such that $\tilde{\varphi}^{m}$ is topologically transitive for all $m \in \mathbf{N}$, then there exists a leftmost-permutive endomorphism $\tilde{\varphi}_{0}$ of the onesided full shift $\left(A^{\mathbf{N}}, \tilde{\sigma}_{A}\right)$ over an alphabet $A$ such that there exists a topological conjugacy $\tilde{\psi}:\left(\tilde{X}_{G^{*}}, \tilde{\sigma}_{G^{*}}\right) \rightarrow\left(A^{\mathbf{N}}, \tilde{\sigma}_{A}\right)$ with $\tilde{\varphi}=\tilde{\psi}^{-1} \tilde{\varphi}_{0} \tilde{\psi}$.

Proof. By Remark 5.2, there exists a onesided 1-1, LL textile system $T^{*}$ over $G^{*}$ with $\tilde{\varphi}_{T^{*}}=\tilde{\varphi}$. Since $\tilde{\xi}_{T^{*}}$ is $1-1$, there exists a factor map (semi-conjugacy) of $\left(\tilde{X}_{T^{*}}, \tilde{\varphi}_{T^{*}}\right)$ onto the onesided woof shift $\left(\tilde{X}_{T}, \tilde{\sigma}_{T}\right)$ of the dual $T$ of $T^{*}$. Therefore, for each $m \in \mathbf{N}$, since $\tilde{\varphi}_{T^{*}}^{m}$ is topologically transitive, so is $\tilde{\sigma}_{T}^{m}$, so that $\sigma_{T}^{m}$ is topologically transitive. Thus the result follows from Proposition 5.1 and Remark 5.2 .

The preceding corollary shows that any (not necessarily expansive) leftmostpermutive or LL endomorphism $\tilde{\varphi}$ of any onesided topological Markov shift such that $\tilde{\varphi}^{m}$ is topologically transitive for all $m \in \mathbf{N}$ is "topologically conjugate" to some leftmost-permutive endomorphism of some onesided full shift (some leftmostpermutive onesided cellular automaton map).

We consider generalizing Theorems 1.1 and 1.3 which concern automorphisms of onesided full shifts (invertible onesided cellular automata) to leftmost-permutive endomorphisms of onesided full shifts (leftmost-permutive onesided cellular automata). As is clear by the proof of Theorem 1.3, the generalization of Theorem 1.3 follows directly from Proposition 3.5. But it will turn out that the whole generalization can be reduced to Theorems 1.1 and 1.3, since every expansive, leftmostpermutive endomorphism of a onesided full shift is necessarily accompanied by expansive automorphisms which have the "same" dynamics as it has. 
Let $\varphi: X \rightarrow X$ be an onto continuous map of a compact metric space with metric $d_{X}$. Let

$$
\mathcal{O}_{\varphi}=\left\{\left(x_{i}\right)_{i \in \mathbf{Z}} \mid \forall i, x_{i} \in X \text { and } \varphi\left(x_{i}\right)=x_{i+1}\right\}
$$

Let $\mathcal{O}_{\varphi}$ be endowed with the metric such that

$$
d_{\mathcal{O}_{\varphi}}\left(\left(x_{i}\right)_{i \in \mathbf{Z}},\left(y_{i}\right)_{i \in \mathbf{Z}}\right)=\sup \left\{2^{-|i|} d_{X}\left(x_{i}, y_{i}\right) \mid i \in \mathbf{Z}\right\} \text {. }
$$

We define a homeomorphism $\sigma_{\varphi}: \mathcal{O}_{\varphi} \rightarrow \mathcal{O}_{\varphi}$ by

$$
\sigma_{\varphi}\left(\left(x_{i}\right)_{i \in \mathbf{Z}}\right)=\left(x_{i+1}\right)_{i \in \mathbf{Z}} \quad\left(x_{i}\right)_{i \in \mathbf{Z}} \in \mathcal{O}_{\varphi} .
$$

Proposition 5.4. Let $A$ be an alphabet. Let $k \in \mathbf{N}$. Let $f: A^{k+1} \rightarrow A$ be a leftmost-permutive local rule. Then for some $l \in \mathbf{N}$, there exists a leftmostpermutive local rule $\hat{f}:\left(A^{2 k}\right)^{l} \rightarrow A^{2 k}$ satisfying the following conditions:

(1) $\tilde{\varphi}_{\hat{f}}:\left(A^{2 k}\right)^{\mathbf{N}} \rightarrow\left(A^{2 k}\right)^{\mathbf{N}}$ is a homeomorphism;

(2) $\tilde{\varphi}_{\hat{f}}$ is expansive if and only if $\tilde{\varphi}_{f}$ is expansive;

(3) if $\tilde{\varphi}_{f}$ is expansive, then $\left(\mathcal{O}_{\tilde{\varphi}_{f}}, \sigma_{\tilde{\varphi}_{f}}\right)$ is topologically conjugate to $\left(\left(A^{2 k}\right)^{\mathbf{N}}, \tilde{\varphi}_{\hat{f}}\right)$.

Proof. Let $T^{*}=\left(p^{*}, q^{*}: \Gamma^{*} \rightarrow G_{A}\right)$ be the textile system in the proof of Theorem 1.3 (Section 4) in which $g$ is such that $\tilde{\varphi}_{g}$ is the identity mapping. Then $\Gamma^{*}=G_{A}^{[k+1]}$, and $p^{*}: \Gamma^{*} \rightarrow G_{A}$ and $q^{*}: \Gamma^{*} \rightarrow G_{A}$ are defined by

$p^{*}\left(a_{1} \ldots a_{k+1}\right)=a_{1}, \quad q^{*}\left(a_{1} \ldots a_{k+1}\right)=f\left(a_{1} \ldots a_{k+1}\right), \quad a_{1} \ldots a_{k+1} \in A_{\Gamma^{*}}=A^{k+1}$.

We have $\tilde{\varphi}_{f}=\tilde{\varphi}_{T^{*}}$. Let $T=\left(p, q: \Gamma \rightarrow G_{B}\right)$ be the dual of $T^{*}$ with $B=A^{k}$. Since $f$ is leftmost-permutive, $T^{*}$ is LL, so that $q$ is biresolving. Let $\bar{T}=\left(\bar{p}, \bar{q}: \bar{\Gamma} \rightarrow G_{B}\right)$ be the $k$-th composition power of $T$. Then $\bar{q}$ is biresolving. By construction, $V_{\bar{\Gamma}}=A^{k}$ and for any arc $\alpha \in A_{\bar{\Gamma}}, i_{\bar{\Gamma}}(\alpha)$ uniquely determines $\bar{p}(\alpha)$; in fact, $i_{\bar{\Gamma}}(\alpha)$ and $\bar{p}(\alpha)$ are the same as an element of $A^{k}$. Hence we define $F: V_{\bar{\Gamma}} \rightarrow B$ by

$$
F\left(i_{\bar{\Gamma}}(\alpha)\right)=\bar{p}(\alpha), \quad \alpha \in A_{\bar{\Gamma}}
$$

Let $\overline{\bar{T}}=\left(\overline{\bar{p}}, \overline{\bar{q}}: \overline{\bar{\Gamma}} \rightarrow G_{B}\right)$ be the second composition power of $\bar{T}$. Then each arc $\gamma$ in $\overline{\bar{\Gamma}}$ is of the form ${ }_{\beta}^{\alpha}$, where $\alpha$ and $\beta$ are $\operatorname{arcs}$ in $\bar{\Gamma}$ with $\bar{q}(\alpha)=\bar{p}(\beta)$. For $\gamma=\begin{gathered}\alpha \\ \beta\end{gathered}$, $\overline{\bar{p}}(\gamma)=\bar{p}(\alpha)$ and $\overline{\bar{q}}(\gamma)=\bar{q}(\beta)$. Since $\bar{q}$ is biresolving, so is $\overline{\bar{q}}$. Let $\hat{T}=\left(\hat{p}, \hat{q}: \hat{\Gamma} \rightarrow G_{B}\right)$ be the textile system such that $\hat{\Gamma}$ and $\hat{q}$ are the same as $\overline{\bar{\Gamma}}$ and $\overline{\bar{q}}$, respectively, but $\hat{p}$ is given as follows. For $\gamma=\frac{\alpha}{\beta}$ in $A_{\hat{\Gamma}}=A_{\overline{\bar{\Gamma}}}$,

$$
\hat{p}(\gamma)=F\left(t_{\bar{\Gamma}}(\alpha)\right)
$$

alternatively $\hat{p}(\gamma)=\overline{\bar{p}}(\delta)$, where $\delta$ is an arc in $\overline{\bar{\Gamma}}$ such that $\gamma \delta$ is a path of length 2 in $\overline{\bar{\Gamma}}$. Then it is observed that $\overline{\bar{T}}$ weaves a half-textile $\left(\gamma_{i, j}\right)_{i \in \mathbf{N}, j \in \mathbf{Z}}$ if and only if $\hat{T}$

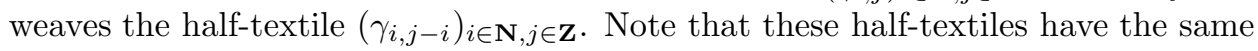
uppermost thread, i.e., $\left(\overline{\bar{p}}\left(\gamma_{1, j}\right)\right)_{j \in \mathbf{Z}}=\left(\hat{p}\left(\gamma_{1, j-1}\right)\right)_{j \in \mathbf{Z}}$. Hence we have

$$
X_{\hat{T}}=X_{\overline{\bar{T}}}=X_{T},
$$

and we see that $\xi_{\hat{T}}$ is $1-1$ if and only if $\xi_{\bar{T}}$ is $1-1$, so that $\xi_{\hat{T}}$ is $1-1$ if and only if $\xi_{T}$ is $1-1$. (If $\xi_{T}$ is $1-1$, then $\varphi_{\hat{T}}=\varphi_{\bar{T}}^{2} \sigma_{\bar{T}}^{-1}$.) We see that $\hat{T}^{*}$ is over $G_{A^{2 k}}$. Since $\hat{q}$ is biresolving, $\hat{T}^{*}$ is LL, so that $\hat{T}^{*}$ is nondegenerate. Hence

$$
\left(\tilde{X}_{\hat{T}^{*}}, \tilde{\sigma}_{\hat{T}^{*}}\right)=\left(\left(A^{2 k}\right)^{\mathbf{N}}, \tilde{\sigma}_{A^{2 k}}\right) .
$$


We shall prove that $\tilde{\xi}_{\hat{T}^{*}}$ and $\tilde{\eta}_{\hat{T}^{*}}$ are bijections. Let $\left(\hat{\gamma}_{i, j}\right)_{i \in \mathbf{N}, j \in \mathbf{Z}}$ be a halftextile woven by $\hat{T}$. Then $\left(\hat{\gamma}_{i, j}\right)_{i \in \mathbf{N}, j \in \mathbf{Z}}=\left(\gamma_{i, j-i}\right)_{i \in \mathbf{N}, j \in \mathbf{Z}}=\left(\begin{array}{c}\alpha_{i, j-i} \\ \beta_{i, j-i}\end{array}\right)_{i \in \mathbf{N}, j \in \mathbf{Z}}$ for a half-textile $\left(\gamma_{i, j}\right)_{i \in \mathbf{N}, j \in \mathbf{Z}}=\left(\begin{array}{c}\alpha_{i, j} \\ \beta_{i, j}\end{array}\right)_{i \in \mathbf{N}, j \in \mathbf{Z}}$ woven by $\overline{\bar{T}}$. It suffices to show that $\left(i_{\hat{\Gamma}}\left(\hat{\gamma}_{i, 0}\right)\right)_{i \in \mathbf{N}}$ uniquely determines $\left(\hat{\gamma}_{i, 0}\right)_{i \in \mathbf{N}}$ and $\left(\hat{\gamma}_{i,-1}\right)_{i \in \mathbf{N}}$. To do this, it suffices to show that $\underset{i_{\hat{\Gamma}}\left(\hat{\gamma}_{2,0}\right)}{i_{\hat{\Gamma}}\left(\hat{\gamma}_{1,0}\right)}$ uniquely determines $\hat{\gamma}_{1,0}$ and $\hat{\gamma}_{1,-1}$. Note that

$$
\hat{\gamma}_{1,0}=\begin{gathered}
\alpha_{1,-1} \\
\beta_{1,-1} \\
\hat{\gamma}_{2,0} \\
\alpha_{2,-2} \\
\beta_{2,-2}
\end{gathered}
$$

where $\alpha_{i,-i}, \beta_{i,-i} \in A_{\bar{\Gamma}}, \bar{q}\left(\alpha_{i,-i}\right)=\bar{p}\left(\beta_{i,-i}\right), i=1,2$, and $F\left(t_{\bar{\Gamma}}\left(\alpha_{2,-2}\right)\right)=\bar{q}\left(\beta_{1,-1}\right)$.

Assume that

$$
\begin{aligned}
& i_{\bar{\Gamma}}\left(\alpha_{1,-1}\right) \\
& i_{\hat{\Gamma}}\left(\hat{\gamma}_{1,0}\right)=i_{\bar{\Gamma}}\left(\beta_{1,-1}\right) \\
& i_{\hat{\Gamma}}\left(\hat{\gamma}_{2,0}\right)=i_{\bar{\Gamma}}\left(\alpha_{2,-2}\right) \\
& i_{\bar{\Gamma}}\left(\beta_{2,-2}\right)
\end{aligned}
$$

is given. Then $i_{\bar{\Gamma}}\left(\beta_{2,-2}\right)$ uniquely determines $F\left(i_{\bar{\Gamma}}\left(\beta_{2,-2}\right)\right)=\bar{p}\left(\beta_{2,-2}\right)=\bar{q}\left(\alpha_{2,-2}\right)$. Since $\bar{q}$ is right resolving, $i_{\bar{\Gamma}}\left(\alpha_{2,-2}\right)$ and $\bar{q}\left(\alpha_{2,-2}\right)$ determine $\alpha_{2,-2}$, so that $t_{\bar{\Gamma}}\left(\alpha_{2,-2}\right)$ is determined. Hence $F\left(t_{\bar{\Gamma}}\left(\alpha_{2,-2}\right)\right)=\bar{q}\left(\beta_{1,-1}\right)$ is determined. Together with $\bar{q}\left(\beta_{1,-1}\right),{ }_{\bar{\Gamma}} i_{\bar{\Gamma}}\left(\alpha_{1,-1}\right)$ determines $\begin{aligned} & \alpha_{1,-1} \\ & \left.\beta_{1,-1}=\beta_{1,0}\right)\end{aligned}$, because $\bar{q}$ is right resolving.

We also see that $i_{\bar{\Gamma}}\left(\alpha_{2,-2}\right)$ determines $F\left(i_{\bar{\Gamma}}\left(\alpha_{2,-2}\right)\right)=\bar{p}\left(\alpha_{2,-2}\right)=\bar{q}\left(\beta_{1,-2}\right)$. To-

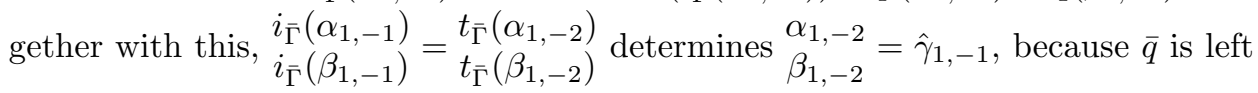
resolving.

Thus we have proved that $\tilde{\xi}_{\hat{T}^{*}}$ and $\tilde{\eta}_{\hat{T}^{*}}$ are bijections. Therefore, $\tilde{\varphi}_{\hat{T}^{*}}=$ $\tilde{\eta}_{\hat{T}^{*}}\left(\tilde{\xi}_{\hat{T}^{*}}\right)^{-1}$ is an automorphism of $\left(\tilde{X}_{\hat{T}^{*}}, \tilde{\sigma}_{\hat{T}^{*}}\right)$. Since $\left(\tilde{X}_{\hat{T}^{*}}, \tilde{\sigma}_{\hat{T}^{*}}\right)=\left(\left(A^{2 k}\right)^{\mathbf{N}}, \tilde{\sigma}_{A^{2 k}}\right)$, there exist $l \in \mathbf{N}$ and a local rule $\hat{f}:\left(A^{2 k}\right)^{l} \rightarrow A^{2 k}$ such that $\tilde{\varphi}_{\hat{f}}=\tilde{\varphi}_{\hat{T}^{*}}$. Since $\xi_{\hat{T}}$ is $1-1$ if and only if $\xi_{T}$ is $1-1, \tilde{\varphi}_{\hat{f}}=\tilde{\varphi}_{\hat{T}^{*}}$ is expansive if and only if $\tilde{\varphi}_{f}=\tilde{\varphi}_{T^{*}}$ is expansive (by [N2, Theorem 2.11(2)]). If $\tilde{\varphi}_{f}$ is expansive, then

$$
\left(\mathcal{O}_{\tilde{\varphi}_{f}}, \sigma_{\tilde{\varphi}_{f}}\right) \cong\left(X_{T}, \sigma_{T}\right)=\left(X_{\hat{T}}, \sigma_{\hat{T}}\right) \cong\left(\left(A^{2 k}\right)^{\mathbf{N}}, \tilde{\varphi}_{\hat{f}}\right)
$$

where $\cong$ denotes topological conjugacy.

Combined with Theorem 1.3 and Proposition 5.4, the theorem of Boyle and Maass (Theorem 1.1) is improved to

Theorem 5.5. Let $A$ be the alphabet of $N$ symbols. Let $f: A^{k+1} \rightarrow A$ be a leftmost-permutive local rule with $k \in \mathbf{N}$. If $\tilde{\varphi}_{f}$ is expansive, then $\left(\mathcal{O}_{\tilde{\varphi}_{f}}, \sigma_{\tilde{\varphi}_{f}}\right)$ is topologically conjugate to a topological Markov shift which is shift equivalent to some full $J$-shift, where $J$ and $N$ are divisible by the same primes.

There exists an expansive, noninvertible, leftmost-permutive onesided cellular automaton map which is not positively expansive. Let $A_{1}$ and $A_{2}$ be alphabets with cardinality not less than 2. Let $f_{1}: A_{1}^{k+1} \rightarrow A_{1}$ be a bipermutive (i.e., leftmost-permutive and rightmost-permutive) local rule with $k \in \mathbf{N}$. Then $\tilde{\varphi}_{f_{1}}$ 
is noninvertible and positively expansive. Let $\tilde{\varphi}_{2}: A_{2}^{\mathbf{N}} \rightarrow A_{2}^{\mathbf{N}}$ be an expansive invertible onesided cellular automaton map, which is not positively expansive by Theorem 3.9 (Schwartzman) in $\left[\mathrm{BL}\right.$. Then the direct product $\tilde{\varphi}_{f_{1}} \times \tilde{\varphi}_{2}$ is an example of the above.

\section{Bipermutive Endomorphisms of mixing topological Markov Shifts}

For integers $k, l \geq 0$, an endomorphism $\varphi$ of a topological Markov shift $\left(X_{G}, \sigma_{G}\right)$ is called a bipermutive endomorphism of $(k, l)$-type if there exists a local rule $f$ : $L_{k+l+1}(G) \rightarrow A_{G}$ which induces a biresolving graph-homomorphism of $G^{[k+l+1]}$ onto $G$ and gives $\varphi$ by

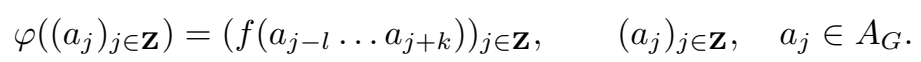

A bipermutive endomorphism of an irreducible topological Markov shift is $N$-toone for some $N \in \mathbf{N}$ (see [N1]). Bipermutive endomorphisms of topological Markov shifts were treated in [N2, Proposition 3.14], but no example has been known for noninvertible bipermutive endomorphisms of a mixing topological Markov shift which is not topologically conjugate to any full shift [N2, p. 52]. By virtue of Proposition 5.1, we can know that there exists no such example.

Proposition 6.1. Let $\left(X_{G}, \sigma_{G}\right)$ be a mixing topological Markov shift having an $N$ to-one bipermutive endomorphism $\varphi$ of $(k, l)$-type with $N \in \mathbf{N}, k, l \geq 0, k+l \geq 1$. Then the following statements are valid.

(1) $\left(X_{G}, \sigma_{G}\right)$ is topologically conjugate to the full $J$-shift with $J^{k+l}=N$.

(2) If $k>0$ and $l>0$, then $\left(X_{G}, \varphi\right)$ is topologically conjugate to the onesided full $N$-shift.

(3) If $k=0$, then $\left(\tilde{X}_{G}, \tilde{\varphi}\right)$ is topologically conjugate to the onesided full $N$ shift, where $\tilde{\varphi}$ is the endomorphism of the onesided topological Markov shift $\left(\tilde{X}_{G}, \tilde{\sigma}_{G}\right)$ naturally induced by $\varphi$.

Proof. Let $f: L_{k+l+1}(G) \rightarrow A_{G}$ be the bipermutive local rule defining $\varphi$. Let $\Gamma=G^{[k+l+1]}$. Let $q: \Gamma \rightarrow G$ be the biresolving graph-homomorphism induced by $f$. Let $T=(p, q: \Gamma \rightarrow G)$ be the textile system with $p$ defined by $p\left(a_{1} \ldots a_{k+l+1}\right)=$ $a_{1}, a_{1} \ldots a_{k+l+1} \in L_{k+l+1}(G), a_{j} \in A_{G}$. Then $T$ is a onesided 1-1 nondegenerate textile system. Clearly $p$ is left resolving and $\tilde{\xi}_{T}$ is $1-1$. Using the property that $q$ is right resolving, we easily see that $\tilde{\xi}_{T^{*}}$ is $1-1$ (see [N2 the proof of Proposition 3.14]). Since $q$ is biresolving, $T^{*}$ is LL. Hence $T^{*}$ is nondegenerate, so that $\left(X_{T^{*}}, \sigma_{T^{*}}\right)$ is a topological Markov shift. Since $\tilde{\xi}_{T^{*}}$ is 1-1, it follows from Theorem 2.12 of [N2] that $\left(\tilde{X}_{T}, \tilde{\varphi}_{T}\right) \cong\left(\tilde{X}_{T^{*}}, \tilde{\sigma}_{T^{*}}\right)$. Since $\tilde{\varphi}_{T}$ is conjugate to a onesided topological Markov shift and commutes with the mixing onesided topological Markov shift $\tilde{\sigma}_{T}$, it follows from Lemma 2.9 of $[\overline{\mathrm{BFF}}]$ or Theorem 1.6 of [N4 that $\tilde{\varphi}_{T}$ is topologically mixing, so that $\tilde{\sigma}_{T^{*}}$ is topologically mixing. Since $T$ is onesided $1-1$ and LL and $\left(\tilde{\sigma}_{T^{*}}\right)^{m}$ is topologically transitive for all $m \in \mathbf{N}$, it follows from Proposition 5.1 that $\left(X_{G}, \sigma_{G}\right)$ is topologically conjugate to the full $J$-shift for some $J \in \mathbf{N}$.

Let $M_{G}$ and $M_{\Gamma}$ be the adjacency matrices of $G$ and $\Gamma$, respectively. Let $l_{0}=\left(l_{0}(u)\right)_{u \in V_{G}}$ and $r_{0}=\left(r_{0}(u)\right)_{u \in V_{G}}$ be left and right eigenvectors of $M_{G}$ corresponding to $J$. Let $l_{1}=\left(l_{1}(v)\right)_{v \in V_{\Gamma}}$ and $r_{1}=\left(r_{1}(v)\right)_{v \in V_{\Gamma}}$ be the row and column vectors such that for $v=a_{1} \ldots a_{k+l} \in V_{\Gamma}=L_{k+l}(G), a_{i} \in A_{\Gamma}$,

$$
l_{1}(v)=l_{0}\left(i_{G}\left(a_{1}\right)\right) \quad \text { and } \quad r_{1}(v)=r_{0}\left(t_{G}\left(a_{k+l}\right)\right) .
$$


Then $l_{1}$ and $r_{1}$ are left and right eigenvectors of $M_{\Gamma}$ corresponding to $J$. We have $l_{1} r_{1}=l_{0} M_{G}^{k+l} r_{0}=J^{k+l} l_{0} r_{0}$. Let $l_{2}=\left(l_{2}(v)\right)_{v \in V_{\Gamma}}$ and $r_{2}=\left(r_{2}(v)\right)_{v \in V_{\Gamma}}$ be the row and column vectors such that $l_{2}(v)=l_{0}\left(q_{V}(v)\right)$ and $r_{2}(v)=r_{0}\left(q_{V}(v)\right)$ for all $v \in V_{\Gamma}$. Then, since $q$ is a biresolving graph-homomorphism with $q_{V} N$-to-one, it follows that $l_{2}$ and $r_{2}$ are left and right eigenvectors of $M_{\Gamma}$ corresponding to $J$ and $l_{2} r_{2}=N l_{0} r_{0}$ (see [N1 Proposition 2.3]). Since $l_{1}$ and $l_{2}$ are eigenvectors of the same irreducible nonnegative matrix corresponding to its spectral radius, we have $l_{1}=c l_{2}$ for some $c>0$. Since $\left\{l_{1}(v) \mid v \in V_{\Gamma}\right\}=\left\{l_{2}(v) \mid v \in V_{\Gamma}\right\}$, we have $c=1$, so that $l_{1}=l_{2}$. Similarly $r_{1}=r_{2}$. Thus we have $N=J^{k+l}$. We have proved (1).

Let $k \geq 0$ and $l>0$. Let $T_{0}=\left(p_{0}, q: \Gamma \rightarrow G\right)$ be the textile system with $p_{0}$ defined by $p_{0}\left(a_{1} \ldots a_{k+l+1}\right)=a_{k+1}, a_{1} \ldots a_{k+l+1} \in L_{k+l+1}(G), a_{j} \in A_{G}$. Then $T_{0}$ is a onesided 1-1 nondegenerate textile system with $\varphi_{T_{0}}=\varphi$. Since $l>0$, it follows that $\tilde{\xi}_{T_{0}^{*}}$ is injective. Hence $T_{0}^{*}$ is a onesided 1-1, LL textile system with $\sigma_{T_{0}}^{m}$ topologically transitive for all $m \in \mathbf{N}$. Therefore, by Proposition 5.1, $\left(\tilde{X}_{T_{0}^{*}}, \tilde{\sigma}_{T_{0}^{*}}\right)$ is topologically conjugate to a onesided full shift. If $T_{0}^{*}$ is over a graph $G^{*}$, then every vertex of $G^{*}$ has exactly $N$ arcs ending in it, because $q_{V}$ is $N$-to-one. Hence $\left(\tilde{X}_{T_{0}^{*}}, \tilde{\sigma}_{T_{0}^{*}}\right)=\left(\tilde{X}_{G^{*}}, \tilde{\sigma}_{G^{*}}\right)$ is topologically conjugate to the onesided full $N$-shift.

If $k>0$, then it follows that $\tilde{\eta}_{T_{0}^{*}}$ is injective, so that we have $\left(X_{T_{0}}, \varphi_{T_{0}}\right) \cong$ $\left(\tilde{X}_{T_{0}^{*}}, \tilde{\sigma}_{T_{0}^{*}}\right)$, by Theorem 2.11 of [N2]. If $k=0$, then, by Theorem 2.12 of [N2], we have $\left(\tilde{X}_{T_{0}}, \tilde{\varphi}_{T_{0}}\right) \cong\left(\tilde{X}_{T_{0}^{*}}, \tilde{\sigma}_{T_{0}^{*}}\right)$. Thus we have proved (2) and (3).

Proposition 6.1 for the case that $\left(X_{G}, \sigma_{G}\right)$ is a full shift (i.e., $G$ is a one-vertex graph) was already known: (2) is found in [SA] and [N2], and (3) is contained in the result in [C] that for a rightmost-permutive endomorphism $\varphi$ of $(0, l)$-type of a full shift $\left(A^{\mathbf{z}}, \sigma_{A}\right)$ with $l \geq 1,\left(A^{\mathbf{N}}, \tilde{\varphi}\right)$ is topologically conjugate to $\left(A^{\mathbf{N}}, \tilde{\sigma}_{A}^{l}\right)$.

In $\mathrm{BM}$, Theorem 9.2], the following result has been given. If an expansive invertible onesided cellular automaton map $\tilde{\varphi}_{f}$ with $f: A^{2} \rightarrow A$ has its inverse $\tilde{\varphi}_{e}$ with $e: A^{2} \rightarrow A$ and if for $a, b \in A, e(a b) a f(a b)$ uniquely determines $b$, then $\left(A^{\mathbf{N}}, \tilde{\varphi}_{f}\right)$ is topologically conjugate to the full $J$-shift with $J^{2}=N$, where $N$ is the cardinality of $A$. In view of Theorem 1.3, Proposition 6.1 can be considered to be an extension of it. For let $T^{*}=\left(p^{*}, q^{*}: G_{A}^{[2]} \rightarrow G_{A}\right)$ be the textile system such that $p^{*}$ and $q^{*}$ are given by $p^{*}\left(a_{1} a_{2}\right)=a_{1}$ and $q^{*}\left(a_{1} a_{2}\right)=f\left(a_{1} a_{2}\right)$ with $a_{1}, a_{2} \in A$. Then the dual $T$ of $T^{*}$ is onesided $1-1,\left(X_{T}, \sigma_{T}\right) \cong\left(A^{\mathbf{N}}, \tilde{\varphi}_{f}\right)$ and $\varphi_{T}$ is an endomorphism of (1,1)-type of $\left(X_{T}, \sigma_{T}\right)$ given by the local rule $e(a, b) a f(a, b) \mapsto b$. By Theorem 1.3 and its proof, $\left(X_{T}, \sigma_{T}\right)$ is topologically conjugate to a mixing topological Markov shift. Hence, for sufficiently large $n,\left(X_{T^{[n]}}, \sigma_{\left.T^{[n]}\right]}\right)=\left(X_{T}^{[n]}, \sigma_{T}^{[n]}\right)$ is a mixing topological Markov shift and it is easily seen that $\varphi_{T^{[n]}}$ is a bipermutive endomorphism of $(1,1)$-type of the shift.

Let an endomorphism $\varphi$ of a topological Markov shift $\left(X_{G}, \sigma_{G}\right)$ be called a $q$-biresolving endomorphism if there exists a onesided 1-1 nondegenerate textile system $T=\left(p_{0}, q_{0}: \Gamma \rightarrow G\right)$ with $q_{0}$ biresolving and $\varphi_{T}=\varphi$. A $q$-biresolving endomorphism of a transitive topological Markov shift is $N$-to-one for some $N \in \mathbf{N}$ (see [N1]). A bipermutive endomorphism of a topological Markov shift is $q$-biresolving. A positively expansive endomorphism $\varphi$ of a transitive topological Markov shift $\left(X_{G}, \sigma_{G}\right)$ is essentially $q$-biresolving, that is, there exist a $q$-biresolving endomorphism $\varphi_{0}$ of a topological Markov shift $\left(X_{G_{0}}, \sigma_{G_{0}}\right)$ and a topological conjugacy $\psi:\left(X_{G}, \sigma_{G}\right) \rightarrow\left(X_{G_{0}}, \sigma_{G_{0}}\right)$ with $\varphi=\psi^{-1} \varphi_{0} \psi$. This follows from Theorem 3.9 of [N2] and the result of Kuka $\mathrm{Ku}$, that a positively expansive endomorphism 
of a transitive topological Markov shift is topologically conjugate to a (surjective) onesided topological Markov shift. (The versions of this and the next result in which the endomorphism is additionally assumed to be onto were obtained independently by the author; see [N3, Section 8].) Combining Kưrka's result and Theorem 3.12 of [N2], we have the result that a positively expansive endomorphism of a mixing topological Markov shift is topologically conjugate to some onesided full $N$-shift. Therefore, the theorem of Boyle and Maass (Theorem 1.1) proves the following. If a mixing topological Markov shift has a positively expansive endomorphism which is $N$-to-one, then the shift is shift equivalent to some full $J$-shift, where $J$ and $N$ are divisible by the same primes. The two conjectures of Boyle and Maass mentioned in Section 1 imply that under the same hypothesis as above, the shift is topologically conjugate to a full shift and $N$ is divisible by $p^{2}$ for every prime $p$ dividing $N$. Proposition 6.1 is consistent with this.

It follows from Proposition 3.5 and Corollary 3.6 that if $T=(p, q: \Gamma \rightarrow G)$ is a onesided 1-1 textile system with $q$ biresolving, $G$ irreducible and aperiodic and $\sigma_{T^{*}}$ topologically mixing, then for some $n \in \mathbf{N}, \varphi_{T^{[n]}}$ is a $q$-biresolving endomorphism of the mixing topological Markov shift $\left(X_{T}^{[n]}, \sigma_{T}^{[n]}\right)$.

Question 6.2. Does there exist a onesided 1-1, nondegenerate, $q$-biresolving textile system $T$ such that $\left(X_{T}, \sigma_{T}\right)$ is not conjugate to any full shift, $\sigma_{T}$ and $\sigma_{T^{*}}$ are topologically mixing, and $\varphi_{T}$ is noninvertible (but not necessarily positively expansive)?

\section{ACKNOWLEDGEMENTS}

In [U] Masaya Uchida made a computer program which generates all local rules $f: A^{2} \rightarrow A$ with $\tilde{\varphi}_{f}$ bijective and $f(a a)=a$ for $a \in A$, and obtained the list of them, where $A$ is the 4 -symbol alphabet. In $\mathrm{Ko}$, Tadashi Kobayashi took away a large part of the pairs $(f, g)$ with $\tilde{\varphi}_{f, g}$ nonexpansive from all the pairs $\left(f: A^{2} \rightarrow A, g: A^{2} \rightarrow A\right)$ with $\tilde{\varphi}_{f}, \tilde{\varphi}_{g}$ bijective (given by using the rules in Uchida's list) and obtained the list of the remaining of relatively small size. Examining some from this was helpful for the author to find the proof of Theorem 1.3.

The author thanks Mike Boyle for a helpful discussion about $q$-biresolving endomorphisms of mixing topological Markov shifts.

\section{REFERENCES}

[AH] N. Aoki and K. Hiraide, Topological Theory of Dynamical Systems, Recent Advances, North-Holland, Amsterdam, 1994. MR 95m:58095

[BFF] M. Boyle, D. Fiebig and U. Fiebig, A dimension group for local homeomorphisms and endomorphisms of onesided shifts of finite type, J. Reine Angew. Math. 487 (1997), 27-59. MR 98i:54020

[BL] M. Boyle and D. Lind, Expansive subdynamics, Trans. Amer. Math. Soc. 349 (1997), 55-102. MR 97d:58115

[BM] M. Boyle and A. Maass, Expansive invertible onesided cellular automata, J. Math. Soc. Japan 52 (2000), 725-740; corrigendum, preprint. MR 2001g:37011

[C] E. M. Coven, Topological entropy of block maps, Proc. Amer. Math. Soc. 78 (1980), 590-594. MR 80m:54055

[F] R. Fischer, Sofic systems and graphs, Monatsh. Math. 80 (1975), 179-186. MR 53:11018

[Ki] B. Kitchens, Symbolic Dynamics, One-sided, Two-sided and Countable State Markov Chains, Springer-Verlag, 1998. MR 98k:58079

[Ko] T. Kobayashi, Computer experiments which investigate the properties of onesided cellular automata (in Japanese), Master's Thesis, Mie University, Tsu (1995). 
$[\mathrm{Ku}] \quad$ P. Kůrka, Languages, equicontinuity and attractors in cellular automata, Ergodic Theory Dynam. Systems 17 (1997), 417-433. MR 98b:58092

[LM] D. Lind and B. Marcus, Symbolic Dynamics and Coding, Cambridge University, 1995. MR 97a:58050

[N1] M. Nasu, Constant-to-one and onto global maps of homomorphisms between strongly connected graphs, Ergodic Theory Dynam. Systems 3 (1983), 387-413. MR 85m:58162

[N2] Textile systems for endomorphisms and automorphisms of the shift, Mem. Amer. Math. Soc. 546 (1995). MR 95i:54051

[N3] Maps in symbolic dynamics, in Lecture Notes of The Tenth KAIST Mathematics Workshop 1995, ed. G. H. Choe, Korea Advanced Institute of Science and Technology, Mathematics Research Center, Taejon, 1996.

[N4] Endomorphisms of expansive systems on compact metric spaces and the pseudoorbit tracing property, Trans. Amer. Math. Soc. 352 (2000), 4731-4757. MR 2001b:54049

[SA] M. A. Shereshevsky and V. S. Afraimovich, Bipermutative cellular automata are topologically conjugate to the one-sided Bernoulli shift, Random Comput. Dynam. 1 (1992), 91-98. MR 93i:58082

[U] M. Uchida, Generation of the leftmost-permutive cellular automata whose global maps are bijective (in Japanese), Master's Thesis, Mie University, Tsu (1994).

Graduate School of Engineering, Hiroshima University, Higashi-Hiroshima 739-8527, JAPAN

E-mail address: nasu@amath.hiroshima-u.ac.jp 